Editorial

\title{
Acknowledgement to Reviewers of Sustainability in 2016
}

\author{
Sustainability Editorial Office \\ MDPI AG, St. Alban-Anlage 66, 4052 Basel, Switzerland; sustainability@mdpi.com \\ Published: 11 January 2017
}

The editors of Sustainability would like to express their sincere gratitude to the following reviewers for assessing manuscripts in 2016.

We greatly appreciate the contribution of expert reviewers, which is crucial to the journal's editorial process. We aim to recognize reviewer contributions through several mechanisms, of which the annual publication of reviewer names is one. Reviewers receive a voucher entitling them to a discount on their next MDPI publication and can download a certificate of recognition directly from our submission system. Additionally, reviewers can sign up to the service Publons (https://publons.com) to receive recognition. Of course, in these initiatives we are careful not to compromise reviewer confidentiality. Many reviewers see their work as a voluntary and often unseen part of their role as researchers. We are grateful to the time reviewers donate to our journals and the contribution they make.

If you are interested in becoming a reviewer for Sustainability, see the link at the bottom of the webpage http://www.mdpi.com/reviewers.

The following reviewed for Sustainability in 2016:

\author{
Abanda, Henry \\ Abastante, Francesca \\ Abaza, Osama A. \\ Abbas, Abdelgadir \\ Abbas, Assad \\ Abdel-Gayed, Ramzy \\ Abdelkefi, Abdessattar \\ Abdel-Salam, Mohamed \\ Abdollahzadeh Jamalabadi, M.Y. \\ Abdulrahman, M.D. \\ Abel, Guy \\ Abid, Muhammad \\ Abou-Senna, Hatem \\ Abraham, Edo \\ Abrate, Graziano \\ Abson, Dave \\ Abu-Reziq, Raed \\ Abuzar, Mohammad \\ Accorsi, Riccardo \\ Acedo, Luis \\ Adam, Anne-Flore \\ Adamides, Emmanuel \\ Adams, Carl \\ Adams, Matthew D. \\ Adams, Matthew P. \\ Adamson, George
}

Adekunle, Timothy O.

Aditjandra, Paulus Teguh

Adivar, Burcu

Adkins, Jeffery

Adler-Nissen, Jens

Agarwal, Sheela

Agliardi, Elettra

Agostini, Lara

Aguaron-Fuente, Elena

Aguejdad, Rahim

Agugliaro, F.M.

Aguilar, Alfredo

Aguilera, Anne

Ahmad, $\mathrm{H}$.

Ahmad, Mushtaque

Ahmadisharaf, Ebrahim

Ahmed, Abdullahi

Ahmed, Ashraf

Ahmed, M. Daud

Ahmed, Rasha M.

Ahn, Changbum

Ahn, Joonmo

Ahn, Young-Hyo

Aibéo Carneiro, M.J.

Aiello, Marco

Airey, David

\author{
Akamani, Kofi \\ Akkerman, Ido \\ Akratos, Christos \\ Aktas, Emel \\ Alamaniotis, Miltiadis \\ Al-Ansari, Tareq \\ Alauddin, Mohammad \\ Albaladejo Pina, Isabel P. \\ Albareda, Laura \\ Albatici, Rossano \\ Albino, Vito \\ Alcantud, José Carlos R. \\ Aldighieri, Barbara \\ Aldrian, Alexia \\ Aledo, Antonio \\ Alexander, Brem \\ Alexander, David \\ Alexander, Peter \\ Alexandridis, Thomas \\ Alfredo, Katherine \\ Alho, André \\ Allahyari, M.S. \\ Allan, Andrew \\ Allen, Melissa R. \\ Allen, Peter M. \\ Allen, Stephen
}


Ally, Moonis R.

Almeida, Manuela

Almeyda Zambrano, A.

Alonso-Almeida, María del Mar

Alrøe, Hugo Fjelsted

Al-Rubaei, Ahmed M.

Alsaaty, Falih M.

Al-Shemmeri, Tariq

Altieri, Miguel

Amado, M. P.

Amado, Miguel P.

Ameer, Rashid

Amelung, Bas

Amor, Cherif

Amor, James David

Amores-Salvadó, Javier

An, Henry

Anand, P.B.

Anastasiou, Eleftherios

Anastopoulos, Ioannis

Andersen, Brannon

Andersen, Otto

Andersen, Rune

Anderson, Faye

Anderson, Molly

Anderson, Randy

Anderson, Randy L.

Anderson, Tim

Andrade, Maria F.

Andrades, Francisco Javier

Andreopoulou, Zacharoula

Andresen, Inger

Andrews-Speed, Philip

Andrieu, Nadine

Angel, James

Angelakis, Andreas

Angelidou, Margarita

Angelis-Dimakis, A.

Angelo, Hillary

Annema, Jan Anne

Annunziata, Azzurra

Anthony, Edward J.

Antikainen, Harri

Antoninka, Anita

Antoniotti, Sylvain

Anttiroiko, Ari-Veikko

Antucheviciene, Jurgita

Antunes, Maria Dulce

Anunciação, Pedro Fernandes

Anvari, Bani

Anwar, Sajid

Anzidei, Marco

Aparicio Baeza, Juan

Apergis, Nicholas
Appolloni, Andrea

Aprile, Maria Carmela

Arato, Miguel

Aravind, Deepa

Arcese, Gabriella

ArchMiller, Althea A.

Arcidiacono, Claudia

Ardi, Romadhani

Ardito, Lorenzo

Ardjmand, Ehsan

Areal, F. J.

Arena, Claudia

Arenas, Alvaro E.

Arent, Douglas J.

Aretano, Roberta

Armani, Andrea

Armbrecht, John

Armenteras-Pascual, Dolors

Arndt, Channing

Arriola, Aitor

Arteaga, Luis

Artmann, Martina

Arvanitidis, Paschalis

Arvidsson, Niklas

Aryana, Saman

Asdrubali, F.

Asioli, Daniele

Asr, Navid Rahbari

Asrari, Arash

Assemi, Behrang

Asteris, Panagiotis G.

Astier, Marta

Asumadu-Sarkodie, S.

Atangana, Alain

Atkinson, Lucy

Atkinson-Palombo, Carol

Atsonios, Konstantinos

Attard, Guillaume

Audette, Gerald F.

Aung, Kendrick T.

Avadí, Angel

Awad, Yaser

Ayalon, Ofira

Ayantunde, Augustine A.

Aydin, Nazli Yonca

Ayoub, Ali

Azadi, Hossein

Azevedo, Américo

Aziz, Muhammad

Bacenetti, Jacopo

Backman, Charles

Backman, Kenneth F.

Bacon, Christopher

Badiger, Shrinivas
Badland, Hannah

Badola, Ruchi

Bae, S. H.

Baek, Joon Sang

Bafna, Sonit

Baghdadi, Fazia

Bahri, Parisa Arabzadeh

Baig, Furqan

Baigorri, Roberto

Bajwa, Naeem

Bajzer, Željko

Bakillah, Mohamed

Bakouros, Ioannis

Bakry, Saad

Bala, Ram

Balaras, Constantinos A.

Baldarelli, Maria Gabriella

Baldassarri, Catia

Baldinelli, Giorgio

Baldwin, James G.

Baležentis, Alvydas

Baležentis, Tomas

Balk, Bert M.

Ballesteros, Rocio

Balthrop, Andrew

Banerjee, Ruman

Banerjee, Simanti

Banfill, Phillip F. G.

Bang, Sungsig

Baños, Carlos Javier

Bansal, Namita

Bao, Shaowu

Barau, Aliyu Salisu

Barba, Virginia

Barberis, Stefano

Barbieri, Jacopo

Barbosa, A. Márcia

Barbosa, Ana Estela

Barca, Emanuele

Bardi, Federica

Bardy, Roland

Barez, Fred

Barghi, Shahzad

Barilla, David

Barjolle, Dominique

Bark, Rosalind H.

Barkenbus, Jack

Barkley, Andrew

Barloková, Danka

Barnes, Frank S.

Barnett, John H.

Barrena, Raquel

Barrett, Garry

Barrett, M.J. 
Barrow, C.J.

Barta, Csengele

Barteczko-Hibbert, Christian

Bartel, Robyn

Bartholomew, Keith

Bartoněk, Dalibor

Basile, Gianpaolo

Bassett, Ellen

Bassett, Scott

Basso, Gianluigi Lo

Basta, Nicholas Thomas

Bastian, Olaf

Basu, Radha

Bathrellos, George D.

Batt, Hussein Ali

Battaglia, Massimo

Battini, Daria

Baudry, Marc

Bauer, Johannes

Baumgardt, Johanna

Baur, Stuart

Bavorová, Miroslava

Bawaneh, Khaled

Bayer, Charlene

Bayer, Peter

Bayle-Sempere, Just

Baynard, Chris W.

Bayram, I. Safak

Beatty, Perrin H.

Bechmann, Marianne

Becker, Bettina

Becker, Randy A.

Becker, Thomas H.

Becker, Till

Beckmann, Markus

Beckmann, Volker

Beevers, Lindsay

Behdad, Sara

Behnood, Ali

Beier, Paul

Beihoff, Bruce

Bell, Andrew R.

Bell, Simon

Bellantuono, Nicola

Bellman, Beryl

Belloni, Elisa

Beltran, Martimez Julian

Belu, Radian

Benadusi, Mara

Benardos, Andreas

Benedetto, Graziella

Benedict, Mark Q.

Ben-Idris, Mohammed

Benin, Samuel
Benner, Maximilian

Bennett, David

Benoît-Moreau, Florence

Benson, Sally M.

Bentes, Isabel

Bentivenga, Mario

Bentley, Yongmei

Berardi, Gigi

Berardi, Umberto

Berbes, Marta

Bereitschaft, Bradley

Berens, Guido

Beretta, Claudio

Berg, Kimberly

Bergdahl, Lars

Berger-Walliser, Gerlinde

Bergheim, Asbjorn

Beritelli, Pietro

Bernard, Scott A.

Bernesson, Sven

Berntzen, Lasse

Berrittella, Maria

Berzi, Lorenzo

Betti, Gianni

Beuchelt, Tina D.

Beveridge, Alim

Bezawada, Ram

Bhandari, Prem B.

Bhattacharya, Mita

Bhattacharyya, Subhes C.

Bhattarai, Keshav

Bhatti, Yasser Ahmad

Bhutta, M. Khurrum S.

Bian, Linkan

Bicocchi, Nicola

Bielefeldt, Angela

Bigazzi, Alexander

Bigelow, Daniel

Bigliardi, Barbara

Bilgihan, Anil

Billington, Corey

Binder, Julia Katharina

Binimelis, Rosa

Binz, Christian

Birch, Kean

Birkved, Morten

Bischoff, Joschka

Bisschop, Lieselot

Biswas, Sagor

Biswas, Wahidul K.

Bithas, Kostas

Biudes, Marcelo

Bjelland, Mark D.

Bjørn, Anders
Black, Iain R.

Black, John

Black, William C.

Blackmore, Chris

Blanchart, Eric

Blanco, Juan

Blanco, Julio

Blanco, Julio Hernández

Blay-Palmer, Alison

Blázquez-Salom, Macià

Bleys, Brent

Blok, Kornelis

Bloom, Dara

Blum, Helcio

Bobinaite, Viktorija

Bobylev, Nikolai

Bocchiola, Daniele

Boer, Dieter

Boesch, Don

Boessen, Adam

Bogenberger, Klaus

Bohnet, Iris C.

Boholm, Max

Boks, Casper

Bolchini, Cristiana

Bolliger, Janine

Bomberg, Mark

Bonaccorso, Brunella

Boncinelli, Fabio

Bonds, Eric

Bonelli, Cristobal

Bonev, Martin

Bonfanti, Angelo

Bonnes, Mirilia

Bonollo, Elisa

Bontje, Marco

Booth, Annie

Booth, Derek

Borger, Tobias

Borgers, Aloys

Borges, Jose

Bornhorst, Theodore J.

Borozan, Đula

Borruso, Giuseppe

Borthwick, Alistair G.L.

Borup, Mads

Borzacchiello, Maria Teresa

Bose, Pablo S.

Bosetti, Luisa

Böske, Janina

Bosman, Rick

Bosomworth, Karyn

Bosselmann, Klaus

Bostater, Charles R. 


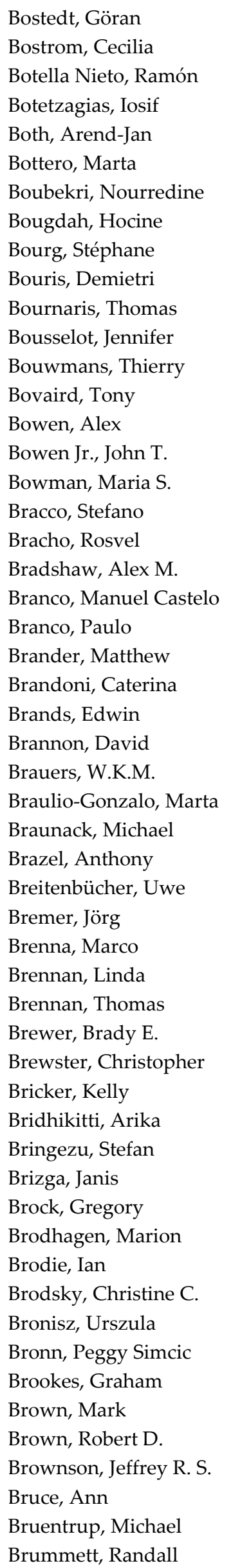

Brunson, Mark

Brussel, Mark

Bruzda, Joanna

Bryant, Benjamin

Bryant, Christopher R.

Bryer, Thomas

Bryson, Douglas

Bucała-Hrabia, Anna

Buchanan, John T.

Buchli, Jürg

Buckley, Alastair

Buckley, Chris

Buenemann, Michaela

Buerke, Anja

Bueyuektahtakin, I. Esra

Buffière, $P$.

Bullón, Teresa

Buratti, Cinzia

Burchart-Korol, Dorota

Bürer, Mary Jean

Burford, Neil

Burkholder, Sean

Burley, Jon Bryan

Burman, Esfandiar

Burnham, Morey

Burrola-Aguilar, Cristina

Burrows, Paul

Burton, Philip

Bussi, Gianbattista

Butler, Leslie (Bees)

Butsic, Van

Butylina, Svetlana

Byeon, Seongjoon

Caballero, Rafael

Cabannes, Yves

Cabiddu, Francesca

Cabrera-Barona, Pablo

Cadet, Frédéric

Cai, Yu

Cairncross, Richard

Cajias, Marcelo

Calautit, John Kaiser

Calderini, Chiara

Calif, Rudy

Callahan, Timothy J.

Callens, Stéphane

Calvo Hernandez, Antonio

Camacho Olmedo, Maria T.

Camarda, Domenico

Camargo, Blanca Alejandra

Cambra-Fierro, Jesus

Cameron, Michael

Cammarata, Kirk

Camões, Aires
Campatelli, Gianni

Campbell, Ben

Campos, Luis Braga

Canavan, Brendan

Cannistraro, Giuseppe

Cánovas, C.R.

Cansino, José M.

Cantarelli, Chantal C.

Cantat, Isabelle

Canto-Perello, Julian

Canu, Donata Melaku

Cao, Kai

Cao, Yang

Capener, C.M.

Capodaglio, Andrea

Capozzoli, Alfonso

Capra, Alessandro

Capurro, Carlotta

Caragliu, Andrea

Caraher, Martin

Cardemil, José M.

Caretta, Martina Angela

Cariñanos, Paloma

Caristi, Giuseppe

Carlisle, Sheena

Carnovale, Steven

Carof, Matthieu

Carpenter-Boggs, Lynne

Carr, Craig

Carranca, Corina

Carreiro, Margaret

Carson, Doris

Carson, Richard T.

Carter, Craig

Cartier, Carolyn

Carvalheiro, L. C.

Carvalho, Ana

Carvalho, Ana Maria

Carver, Steve

Casagrande, Marion

Case, Sean

Caselli, Marco

Casello, Jeffrey M.

Cassetti, Gabriele

Castellani, Beatrice

Castellani, Davide

Castells-Quintana, David

Castilla, Maria

Castro, A. Peter

Castro-Nuño, Mercedes

Castro-Santos, Laura

Catalina, Tiberiu

Cats, Oded

Causevic, Senija 
Cavani, Luciano

Cavicchi, Alessio

Cayzer, Steve

Cerdá, Artemio

Cerini, Diana

Cerreta, Maria

Certomà, Chiara

Cerutti, Alessandro K.

Cervellini, Marco

Cesaroni, Fabrizio

Cetin, Kristen S.

Chabay, Ilan

Chaffin, Brian

Chaganti, Vijayasatya N.

Chalker-Scott, Linda

Chamberlain, Jim

Chan, A.L.S.

Chan, Edwin

Chan, Pak Wai

Chan, Yu-Wei

Chang, Chun Ping

Chang, Hung-hao

Chang, I-Chun Catherine

Chang, Kaowen

Chang, Ming-Chung

Chang, Ping Hung

Chang, Sheng-Hsiung

Chang, Shu-Chen

Chang, Tian-Pau

Chang, Tsangyao

Chang, Yi

Chapman, Jamie

Chappell, Jahi

Chaput, Gina

Charter, Martin

Chatzidiakou, Lia

Chau, K.W.

Chau, Yuen

Chaudhary, Abhishek

Chaudhry, Sohail S.

Chaudy, Yaëlle

Che, Weitao

Chelleri, Lorenzo

Chembo, Yanne K.

Chen, Bin

Chen, Chien Min

Chen, Chun

Chen, Duan

Chen, GuoQian

Chen, James K.C.

Chen, Jun

Chen, Kongzhe

Chen, Lei

Chen, Liangbiao
Chen, Nai-Hua

Chen, Rachel J.C.

Chen, Shih-Chih

Chen, Shyi-Ming

Chen, Tao

Chen, Vincent Yiu-por

Chen, Wan-Jiun

Chen, Wendy Y.

Chen, Yi-Min

Chen, Yiqun

Chen, Yong

Chen, Yunguang

Cheng, Chingwen

Cheng, Enjiang

Cheng, Linlin

Cheng, Xiaohui

Cheng, Y.S.

Cheon, SangHyun

Cherp, Aleh

Cheshmehzangi, Ali

Cheung, Lewis T.O.

Cheung, Wai Ming

Chhajed, Dilip

Chiang, Chung-Yean

Chiarini, Andrea

Chien, Shiuh-Shen

Chifos, Carla

Chikumbo, Oliver

Chikweche, Tendai

Childers, Dan

Chileshe, Nicholas

Chin, Jae Teuk

Chiou, Shang-chia

Chiu, Chung - Yi

Chiu, Yi-Bin

Chiu, Yi-Wen

Chiu, Yung-ho

Chmutina, Ksenia

Cho, Kyu Taek

Cho, Young I.

Choi, Beomjoon

Choi, Dong Gu

Choi, Hojong

Choi, Hyoung Jin

Choi, Jong-Seo

Choi, Sangdo (Sam)

Choi, Sungyong

Choi, Tsan-Ming

Choi, Yongrok

Choi, Yosoon

Chong, Heap-Yih

Christakos, George

Christaras, Basile

Christian-Smith, Juliet
Christofi, Andreas C.

Christoforou, Asimina

Christophe, Vial

Christy, B. P.

Chrpa, Lukas

Chu, Chih-Peng

Chuang, Abel

Chuang, Tsai-Fu

Chudnovsky, Alexandra

Chung, Chun-Jen

Chung, Jacky

Chung, Jin-Do

Chung, Kung-ming

Chung, Siu Wai William

Ciabattoni, Lucio

Cialani, Catia

Ciarkowska, Krystyna

Cicatiello, Clara

Cihacek, Larry

Ciolli, Marco

Cipollina, Maria

Ciraolo, Luigi

Ciriello, Valentina

Ciriminna, Rosaria

Cismaru, Magdalena

Ciuffo, Biagio

Clark, Nigel

Clarke, Paul

Clarkson, Peter M.

Clavel, Danièle

Cliff, David

Clifton, Judith

Coccossis, Harry

Cockrill, Antje

Coelho, Dulce

Coenen, Lars

Coffman, Makena

Cohen, Matthew

Cohen, Maurie J.

Cole, Christine

Cole, Stroma

Coleman, Gill

Colinet, Gilles

Colla, Valentina

Colli, Andrea

Collier, Kevin J.

Collins, John

Collins, LaPorchia

Collins, M. Robin

Colson, Greg

Coma, Marta

Comarazamy, Daniel

Comas, Jordi

Comber, Rob 
Comeig, Irene

Comi, Antonio

Cong, Ronggang

Congedo, Paolo Maria

Conlon, Kathryn C.

Conner, David

Constance, Douglas

Conte, Emilia

Contestabile, Pasquale

Conticelli, Elisa

Contini, Daniele

Contreras, Sergio

Convertino, Matteo

Cookey, Peter

Cools, Mario

Cooper, Ricky

Copeland, Mary Kay

Corbelle Rico, Eduardo Jose

Cordera, Rubén

Cori, Liliana

Corkery, Linda

Cornell, Sarah

Corsi, Stefano

Corsini, Filippo

Cortignani, Raffaele

Coscieme, Luca

Costa, e Inês

Costello, Christine

Costola, Michele

Cotton, Deborah

Couch, Robert

Coulter, John

Courvisanos, Jerry

Cowling, Marc

Cox, Amanda

Cox, Linda J.

Cox, Peter

Coyne, Mark

Cozzi, Mario

Cramer, Jacqueline

Crescimanno, Maria

Cropper, Paul

Cross, Michael

Cruz, Mary Jane Dela

Csereklyei, Zsuzsanna

Cuffaro, Nadia

Cui, Lizhen

Cullinane, Kevin

Cunningham-Sabot, Emmanuele

Cuomo, Sabatino

Curiel-Esparza, Jorge

Curtis, Chadwick C.

Cusick, John

Custódio, Danilo
Custódio, Luísa

Cutler, Robert M.

Czimczik, Claudia I.

D'Andrea, Antonio

D’Elia, Alfredo

Dablanc, Laetitia

D'Adamo, Idiano

Daddi, Tiberio

Dagoumas, Athanasios

Dai, Hanchen

Daim, Tugrul

Dakpo, K Hervé

Dal Maso, Lorenzo

Dalal, Ram

Dale, Ann

Dale, Crispin

Dale, Gareth

Dalton, Alice M.

Dalton, Christina M.

Damette, Olivier

D'Amico, Mario

Dana, Léo-Paul

Dangelico, Rosa Maria

Dangi, Mohan B.

Dani, Samir

Daniels, Tom

Darbra, R.M.

Darbra, Rosa-mari

Darby, Kate

Dare, Melanie (Lain)

Darques, Régis

Dasgupta, Shouro

Dasgupta, Utteeyo

Daskalopoulou, Irene

Dassenakis, Manos J.

Davidson, Brian

Davidson, Marc D.

Davies, Chantel

Davies, Linda

Davies, Thomas G. Emyr

Davis, Kyle

Davis, Mary

Davlasheridze, Meri

Dawidowicz, Agnieszka

Dawson, David A.

Day, Jonathon

De Almeida, António Campar

De Andres, Adrian

De Arroyabe, J.C.F.

De Brito, Jorge

De Feo, Giovanni

De Gennaro, Gianluigi

De Giacomo, Maria Rosa

De Giovanni, Pietro
De Gisi, Sabino

De Gobbi, Maria Sabrina

De Godos, Ignacio

De Gruyter, Christopher

De Hoces, Mónica Calero

De Jong, Eline

De Jong, Martin

De Jong, Wil

De Kervenoael, Ronan

De La Barre, Suzanne

De Lieto Vollaro, Andrea

De Melo, Tadeu C. Cordeiro

De Mendoza, F.P.

De Meo, Emilio

De Miguel, Angel

De Molina, Manuel Gonzalez

De Nicola, Antonio

De Paola, Francesco

De Pelsmacker, Patrick

De Preux, Laure B.

De Schepper, Guillaume

De Stefano, Lucia

De Vries, Lisette

Deakin, Mark

Deal, Brian

Deale, Cynthia S.

Dean, Lorraine T.

Decker, Ryan

Degefie, D.T.

Dehkordi, S. Emad

Deitch, Matthew J.

Deksissa, Tolessa

Del Barrio, Gabriel

Del Brío, Jesús Ángel

Del Pero, Claudio

Delgado, Alexi

Delgado, Jorge

Delgado-Ceballos, Javier

Della Spina, Lucia

Dell'Amico, Mauro

Demartini, Paola

Demissie, M.G.

Den Uyl, Roos M.

Deng, Yong

Denis, Eric

Dennis, Charles

Dentchev, Nikolay

Dentoni, Domenico

Deo, Randhir

Dergiades, Theologos

Derigent, William

Derkzen, Marthe L.

Deryng, Delphine

Despoudi, Stella (Styliani) 
Deutsch, Jonathan

Devika, Kannan

Dewan, Ashraf

Dewil, Raf

Dhanda, Kathy

Dhir, Sanjiv

Dhital, Narayan

Di Gravio, Giulio

Di Battista, Davide

Di Gioia, Maria Luisa

Di Maio, Francesco

Di Maria, Eleonora

Di Palma, Luca

Dias, Ana Cláudia

Díaz, Rolando

Diaz-Balteiro, Luis

Diaz-Espejo, Antonio

Dick, Jan

Diderichsen, Finn

Diener, Derek L.

Díez-Vial, Isabel

Dijk, J.P. (Jitse) Van

Dimas, A. A.

Dimech, Mark

Dimitriou, Dimitrios

Dimitriou, Elias

Dimitrova, Elena

Dimpfl, Thomas

Dinis, Isabel

Dinkel, Danae

Dionisi, Davide

Dittmar, Michael

Divigalpitiya, Prasanna

Dixit, Manish K.

Dixon, Alan

Dixon, Mark

Dizdaroglu, Didem

Djanibekov, Utkur

Do, $\mathrm{M}$.

Do, Ton Duc

Do Paço, Arminda

Dobbs, Cynnamon

Dobre, Ciprian

Dodds, Rachel

Doglioni, Angelo

Doherty, James M.

Dolan, Dale

Doley, David

Dolinsky, Martin

Dolores Esteban, M.

Domazet, Mladen

Domenech, Bruno

Domenech, Teresa

Dominguez Dafauce, L.C.
Domínguez-Navarro, J.A.

Domopoulou, Asimina E.

Donateo, Teresa

Done, James

Dong, Huijuan

Dong, Liang

Dong, Xiaobin

Donia, Enrica

Donnet, Timothy

Dooley, Kevin J.

Dora, Manoj

Dörgeloh, Werner

Döring, Thomas

Dougherty, Michael John

Dovì, Vincenzo

Dreber, Niels

Drezner, Noah D.

Driml, Sally

Droste, Arjan

Drouiche, Nadjib

D'Souza, Clare

Du Plessis, Chrisna

Duffy, Lauren

Dumortier, Jerome

Duncan, Walter M.

Dunn, Ryan J. K.

Dunning, Rebecca

Duppati, Geeta

Dur, Fatih

Duram, Leslie

Đurasević, Mirjana

Duren, Iris Van

Durner, Edward Francis

Duro, Juan Antonio

Dutt, Ashok K.

Duvic-Paoli, Leslie-Anne

Dwivedi, Dipankar

Dwivedi, Puneet

Dworkin, Seth

Eberle, Ulrike

Eble, Cortland F.

Ebner, Jacqueline

Eckerd, Stephanie

Eden, Susanna

Edraki, Mansour

Eeckels, Bruno

Egan, Kevin J.

Egermann, Markus

Ehmke, Jan Fabian

Ehrhardt, Kyle

Ehrig, Hans-jurgen

Ehsani, Mehrdad

Eik, Lars Olav

Eisenegger, Mark
Ekanayake, E. M.

Ekonomou, Lambros

El Ghoul, Sadok

Elahi, Behin

Eleftheriadis, Iordanis M.

Elle, Morten

Ellinger, Andrea D.

Ellis, Eugenia Victoria

Elsanabary, M.H.

Elving, Wim

Emadodin, Iraj

Emmanuel, Kakaras

Emrich, Christopher T.

Endress, Bryan A.

Engelbrecht, Hans-Jurgen

Engle, Nathan L.

Epelde, Lur

Eppink, Florian V.

Erdi, Peter

Erickson, Larry

Ernst, Mathias

Escalera-Reyes, Javier

Escrig-Olmedo, Elena

Espelta, Josep M.

Espino-Rodríguez, Tomás F.

Espionaza, Omar

Esposito, Emilio

Essah, Emmanuel A.

Estes Jr., Maurice G.

Etheridge, Randall

Eusébio, Celeste

Evangelou, M.W.

Evans, James

Everaert, Kim

Eyer, Jonathan

Fabbri, Kristian

Fabricatore, Carlo

Facca-Miess, Tina

Facchinetti, Davide

Facchini, Angelo

Fagence, Michael

Fagerstrøm, Asle

Fagin, Todd

Fahmi, Fikri Zul

Fakhr, Mohamed

Falavigna, Greta

Falcocchio, John C.

Falcone, James A.

Falconer, Lynne

Falk, Martin

Fallmann, Joachim

Fam, Dena

Fancello, Gianfranco

Fanelli, Viviana 
Farah, Paolo

Farahmand, Kambiz

Fargnoli, Mario

Farhadzadeh, Ali

Farmer, Jenny

Farnsworth, Derek

Fassam, Liam

Fath, Brian

Fathi, Mohammad Reza

Fatimah, Yun Arifatul

Faulin, Javier

Faustino, Pedro

Favuzza, Salvatore

Fawcett, Stanley E.

Fayazbakhsh, M.A.

Fayoumi, Amjad

Faysse, N.

Feher, Alexander

Feijoo, Gumersindo

Feiock, Richard

Feketeová, Zuzana

Felice, Emanuele

Felisberto, Paulo

Feng, Wei

Feng, Wen

Feng, Wenting

Feng, Xuehao

Fereidouni, Alireza

Fergen, Joshua

Ferguson, Keith

Feria Toribio, José María

Fernandes, J.A.

Fernandes, Paulo

Fernández García, F.J.

Fernandez-Feijoo, Belen

Fernández-Ramírez, L.M.

Fernando, Ana Luisa

Ferracuti, Francesco

Ferrara, R. M.

Ferrari, Sonia

Ferrario, M.F.

Ferrell, Jeremy

Ferrero, Enrico

Ferretti, Valentina

Ferri, Francesco

Festa, Demetrio Carmine

Fick, Jerker

Ficko, Andrej

Fidel, Rivka

Fierro, Angelo

Figueroa-Armijos, Maria

Filer, Randall K.

Filgueira, Ramón

Filimonau, Viachaslau
Filion, Pierre

Filipponi, Mirko

Filler, Günther

Finnveden, Göran

Fiore, Silvia

Fiorito, Francesco

Firbank, Les

Fischer, Matthias

Fischer, Thomas

Fisher, Joshua

Fisher, Ron

Fistola, Romano

Fitjar, Rune Dahl

Fitzgibbon, John

Fitzpatrick, Patricia

Flapper, Simme Douwe

Flaten, Ola

Fletcher, Luke

Florea, Liviu

Florio, Gaetano

Fluharty, David

Foereid, Bente

Fontana, G.

Fonte, Maria

Foraboschi, Paolo

Foran, Tira

Forbes, Vivian Louis

Forciniti, Carmen

Ford, W. Randolph

Forrester, William

Fortuna, Luigi

Foudi, Sebastien

Foulds, Chris

Fountain, Michael W.

Fourie, Andy

Fowler, Cissy

Fragomeni, Sam

Francis, Charles

Franco, Alessandro

Franco, Maria

Frank, Bjoern

Franklin, Alex

Franklin, Dorcas H.

Frans, Van Geer

Fransen, Koos

Fraszczyk, Anna

Fratino, Umberto

Frayret, Jean-Marc

Frazier, Amy E.

Freedman, Jeff

Frelich, Lee

Frey, Marco

Freybote, Julia

Freymueller, Jeff
Friar, John H.

Friedman, $\mathrm{H}$.

Friend, Mark A.

Frizelle, Gerry

Fry, Matthew

Fu, Tengfei

$\mathrm{Fu}$, Xin

Fuchs, Gerhard

Fuentes, Christian

Fuinhas, José Alberto

Fujibe, Fumiaki

Fujii, Hidemichi

Fujita, Kohei

Fujita, Toshiyuki

Fullen, Michael

Fung, Jimmy

Fürst, Christine

Gabbar, Hossam A.

Gabbert, Silke

Gadanakis, Yiorgos

Gadotti, Alessia

Gagnon, Yves

Gai, Yunwei

Galanakis, Charis M.

Galati, Francesco

Gall, Melanie

Gallego, Alejandro

Gallo, Maria

Gallo, Michela

Galloppo, Giuseppe

Gallos, Antoine

Galpin, Timothy

Galvão, João Rafael

Galvin, Raymond

Gamberini, Rita

Gamborg, Christian

Gan, Muye

Ganippa, L.C.

Gao, Jungang

Gao, Peng

Gao, Xiangpeng

Gao, Xiaolu

Gao, Yingzhi

Gao, Zhen

Gaoue, Orou G.

Garau, Chiara

Garavaglia, Christian

Garay, Luis

Garcia, Ricardo

Garcia, Rita

García, Carmelo R.

García Del Amo, David

García Díaz, Pilar

Garcia Fraile, Paula 
Garcia Melon, Monica

García Navarro, Justo

García-Álvarez, M.T.

García-Arca, Jesús

García-Cascales, M.S.

García-Diego, F.J.

García-Frapolli, Eduardo

García-Segura, Tatiana

García-Taengua, Emili

Garcia-Triviño, Pablo

Gari, Sirak Robele

Garmsiri, Shahryar

Garnett, Kenisha

Garnevska, Elena

Garone, Philip

Garrick, Norman W.

Garrone, Paola

Garyfallos, Arabatzis

Garza-Rodriguez, Jorge

Gasparon, Massimo

Gatzia, Dimitria

Gaunaa, Mac

Gawronski, Stanislaw

Gayle, George-Levi

Gázquez-Abad, J.C.

Gebelein, Jennifer

Geberemariam, T.K.

Gebhardt, Matthew

Geenen, Sara

Geijzendorffer, Ilse

Geißdörfer, Martin

Gelbmann, Ulrike

Gelegenis, John

Gentle, Popular

Gentry, James W.

Gentry, Randall W.

Geoghegan, Hilary

Georgakellos, Dimitrios A.

Georgiadis, Charalambos

Georgiadis, Patroklos

Georgilakis, Pavlos S.

Gerbault, Pascale

Gerend, Jennifer

German, Laura

Gerster-Bentaya, Maria

Geyer, Philipp

Ghafari, Ehsan

Ghazi-Nezami, Farnaz

Gherghina, Stefan Cristian

Ghezehei, Solomon

Ghinea, Cristina

Ghisellini, Patrizia

Ghisetti, Claudia

Ghorbani, Abdolbaset
Ghorbani, Yousef

Ghosh, Prasanta K.

Giannaros, Theodore M.

Gianni, Maria

Giannis, Apostolos

Gibney, Raymond

Gibson, Huston

Gibson, John

Giergiczny, Marek

Giese, Marcus

Giger, Markus

Gil, Emilio

Gilart, Virgilio

Gill, Harsimran K.

Gillam, Erin H.

Gillespie, Josephine

Gilmer, Alan

Giorgio, Guariso

Giraldo, Mario

Girardet, Xavier

Girardin, Eric

Girling, Robert

Gislason, Maya

Gissey, G.C.

Giudice, Fabio

Giudici, Giancarlo

Giuffrida, Alessandra

Giuffrida, Salvatore

Giuliani, Diego

Giuseppe, Genon

Giustiniano, Luca

Givens, Jennifer

Gkiougkis, Ioannis

Glass, Christopher

Glenna, Lealand L.

Glöser, Simon

Glowacka, Katarzyna

Glynn, James

Glynn, Peter J.

Gober, Patricia

Göçmen, Aslıgül

Godos-Díez, José-Luis

Goeppert, Nadine

Goetz, Stephan J.

Goggins, William B.

Goh, Mark

Goh, Yee Mey

Goharian, Erfan

Gohlke, Julia

Golasi, Iacopo

Golinska-Dawson, Paulina

Gollagher, Margaret

Golovina, Svetlana

Golshani, Amir
Gomes, Rui

Gómez-Bezares, F.J.

Gómez-Calvet, Roberto

Gomez-Conde, Jacobo

Gómez-Gutiérrez, Álvaro

Gonela, Vinay

Gong, Chongfeng

Gonzalez, Ainhoa

Gonzalez, E.D.R.S.

González Fidalgo, E.

González-Eguino, M.

González-Velasco, C.

Goodin, Douglas G.

Gorai, Amit

Gordon, Cameron

Gordon, Sean

Gordon, Steven I.

Görg, Christoph

Görgens, Johann F.

Gorgitano, Maria Teresa

Gori, Elena

Gornitz, V.

Gosling, Louisa

Gotsch, Matthias

Gottschalk, Klaus-Günter

Gøtze, John

Gou, Zhonghua

Gourbesville, Philippe

Govindan, Kannan

Gradinaru, Simona Raluca

Graditi, Giorgio

Grafakos, Stelios

Grafius, Darren

Grafton, Miles

Grainger, Stephen

Gralla, Fabienne

Granados, Jesus

Granie, Marie-Axelle

Grant, Jonathan

Grassi, Ausilia

Greaves, Stephen P.

Greco, Marco

Gregg, Jay Sterling

Grekousis, George

Grieshop, James

Griffin, Robert

Grilli, Gianluca

Grillone, Giovanni

Grimaldi, Michele

Grissemann, Ursula S.

Grobman, Yasha Jacob

Grönman, Kaisa

Groom, Geoff

Gross-Camp, Nicole 
Grossman, Jake J.

Growe, Anna

Grundmann, Reiner

$\mathrm{Gu}$, Ning

Guarini, Enrico

Guerreiro, Sérgio

Guerrieri, Marco

Guesmi, Khaled

Guevara, C. Angelo

Gugerell, Katharina

Gugliermetti, Franco

Guido, Giuseppe

Guidolin, Mariangela

Guillen, Jordi

Guillou, Nicolas

Gunawan, Indra

Güneralp, Burak

Guo, Ping

Guran, Serpil

Gurung, Thulo Ram

Gustafsson, Peter

Gustavsson, Eva

Gustavsson, Matthias

Gutierrez, Antonio

Guyot, Alexis

Guziana, Bozena

H Schulze, Christian

Ha, Hoehun

Haack, Barry

Haapala, Antti

Haas, Willi

Haase, Diane

Hacklin, Fredrik

Hafeznezami, Saeedreza

Hafkesbrink, Joachim

Hagens, Janneke E.

Hagens, Nate

Hahn, Rüdiger

Haight, Joel M.

Hair, Joseph F.

Hájek, Petr

Halde, Caroline

Haldi, Frédéric

Hale, James

Halevi, Gali

Halevidis, Constantinos D.

Halfield, Jerry

Halik, Ümüt

Hall, C. Michael

Hall, Damon M.

Hall, Monika

Hall, Murray R.

Haller, Andreas

Halleux, Jean-Marie
Hamiduddin, Iqbal

Hamilton, A.C.

Hamilton, Timothy

Hamm, Michael

Hammar, Linus

Hamstead, Zoe

Han, SangUk

Hansson, Anders

Hansson, Helena

Haq, Noorul

Haque, Mahfuzul

Haramoto, Erin

Harder, Marie

Harder, Robin

Harding, Susan-Marie

Hardman, Michael

Hardy, Anne

Hargadon, Andrew B.

Hargroves, Charlie

Harrington, Julie

Harris, Fiona

Harris, J. Roger

Harrison, Kevin

Harrison, Roy

Hartmann, Matthias

Hartmann, Timo

Hartung, Frank

Harvolk-Schoening, Sarah

Harwood, Ian

Hasan, Reaz

Hasan, Samiul

Hasanov, Akram S.

Hassan, Gazi M.

Hassink, Jan

Hasu, Mervi

Hatziadoniu, Constantine

Hatzichristos, Thomas

Hauptmanns, Ulrich

Hawken, Scott

Hawkes, Andrea

Hawkins, Peter

Haworth, James

Hayakawa, Kazuichi

Hayakawa, Kazunobu

Hayashi, Masaki

He, Gang

He, Guizhen

He, Jibin

He, Jun

He, Mengchang

He, Zhongqi

Headlee, William

Hebner, Robert

Heckenberger, Michael J.
Heckman, Joseph

Heckmann, Tobias

Heerink, Nico

Hegger, Dries

Heidarinejad, Mohammad

Heijman, W.J.M.

Heijungs, Reinout

Heilbrunn, Sibylle

Heindl, Peter

Heinimann, Hans

Heinonen, Jukka

Heinonen-Tanski, Helvi

Heinrichs, Harald

Hellin, Jon

Helmers, Christian

Helmreich, Brigitte

Helms, Marilyn Michelle

Hemmes, Kas

Henderson, Andrew

Henjewele, Christian

Henkin, Zalmen

Hennig, Benjamin D.

Henninger, Claudia

Henriques, C. Oliveira

Henseler, Martin

Hensgen, Frank

Henthorne, Tony

Her, Younggu

Heras-Saizarbitoria, Iñaki

Herczeg, Gabor

Hernandez Garcia, M.T.

Hernandez-Munoz, Luis

Heron, Graeme

Herr, Joshua R.

Herrera-Melián, José Alberto

Herrero, Hannah

Herrmann, Dustin

Herrmann, Dustin L.

Heurtebise, Jean Yves

Heyvaert, Alan C.

Higgins, Vaughan

Higgins-Desbiolles, Freya

Higham, James

Hilbert, Martin

Hildebrandt, Torsten

Hill, Callum

Hill, Douglas

Hille, Stefanie

Hillier, Jonathan

Hilton, Brian N.

Hilty, Lorenz

Hinostroza, Miriam L.

Hinrichs, Christian

Hintermann, Beat 
Hirano, Y.

Hirooka, K.

Hirschberg, Stefan

Hirst, Peter

Hirt, Sonia

Hjelmervik, Jon

Hjorth, Peder

Ho, Ching-Hsiang

Ho, Hung Chak

Ho, Jyh-Wen

Ho, Kevin K.W.

Ho, Shu-Yi

Hoa, Tran Van

Hobson, Kersty

Hochrainer-Stigler, Stefan

Hodgett, Richard

Hoekstra, Rutger

Hoff, Craig J.

Hoffmann, Sabine

Högberg, Lovisa

Holderbaum, William

Holl, Adelheid

Holladay, Patrick

Holland, J.M.

Holland, Michael

Holländer, R.

Höller, Stefanie

Holliday, Lisa

Holopainen, Riikka

Holroyd, Carin

Höltinger, Stefan

Holvad, Torben

Hong, I-Hsuan

Hong, Taehoon

Hong, Wei-Chiang

Honjo, Tsuyoshi

Honkanen, Antti

Hoogervorst, Jan A. P.

Hoornweg, Daniel

Hooshyar, Milad

Hope, Alex

Hopkins, Debbie

Hoppe, Thomas

Hoque, Shamia

Horng, Jeou-Shyan

Horowitz, Leah S.

Horst, Megan

Horváth, Imre

Hospido, Almudena

Hossain, A.K.M. Azad

Hossain, Iqbal

Hossain, Md. Sarwar

Hotta, Yasuhiko

Hottenrott, Hanna
Hou, Jian

Houghton, Tom

Houng, Harvey J.

Howard, Jeffrey L.

Howard, Peter H.

Howard, Philip H.

Howes, Michael

Howlader, Abdul Motin

Hrebicek, Jiri

Hseu, Zeng-Yei

Hsiao, Bo

Hsiao, Chun-Ching

Hsieh, Ying-Che

Hsu, Angel

Hsu, Chia-Lin

Hsu, Ming-wei

Hsu, Ping

$\mathrm{Hsu}$, Ting-Yu

$\mathrm{Hu}$, Allen $\mathrm{H}$.

$\mathrm{Hu}$, Hui

$\mathrm{Hu}$, Liming

$\mathrm{Hu}$, Mingming

$\mathrm{Hu}$, Yingjie

$\mathrm{Hu}$, Zhiyong

$\mathrm{Hu}$, Zhiyong

Huan, Tzung-Cheng

Huang, Cho-Ying

Huang, Gongsheng

Huang, Gordon

Huang, Guo H.

Huang, Guohe

Huang, Huiping

Huang, Kuo-Feng

Huang, Qian

Huang, Qingxu

Huang, Shu-Wei

Huang, Te-Sheng

Huang, Tianyu

Huang, Wu-jang

Huang, Yinlun

Huang, Yue

Huber, Franz K.

Hübner, Renate

Huettmann, Falk

Hugé, Jean

Hughes, Gordon A.

Hughes, Llewelyn

Hughs, Sara

Hung, Yung-Tse

Hunt, Dexter V.L.

Huq, Nazmul

Hurtubia, Ricardo

Huston, Simon

Hutchinson, Mark
Hwang, Bon-Gang

Hwang, Heasoo

Hwang, Ruey-Lung

Hynes, Michael

Iacovidou, Eleni

Iannazzo, Daniela

Ibáñez Mendizábal, Raquel

Ibrahim, Nadine

Idowu, O. John

Idris, Ahmed

Iiames, John S.

Ikerd, John E.

Iliadis, Lazaros

Ilieva, Rositsa

Imeson, Anton

Immitzer, Markus

Inard, Christian

Inazumi, Shinya

Ince, David

Indirli, Maurizio

Infante Amate, Juan

Ingram, Lachlan

Inman, R. Anthony

Inostroza, Luis

Ioannides, Dimitri

Irawan, Agus Bambang

Irger, Matthias

Isaak, Robert

Isabelle, Muller

Isaksson, Raine

Iseki, Kohtaro

Ishizaka, Alessio

Islam, Syed

Ito, Takuya

Ivanova, Maria

Iwu, Chux Gervase

Jabareen, Yosef

Jabłoński, Adam

Jabłoński, Marek

Jackson, Elizabeth

Jackson, Susan E.

Jacob, Rhys

Jacobs, Katharine L.

Jacobs-Crisioni, Chris

Jadidi Mardkheh, A. (Mojgan)

Jaganathan, Arun

Jäger-Waldau, Arnulf

Jain, Manisha

Jain, Mohan Shri

James, Harvey S.

Janda, Karel

Jandl, Robert

Janes, Robert R.

Jang, Ho Won 
Jánosi, Imre

Jansson, Johan

Jardon, Carlos M.

Järveläinen, Jonna

Jarvis, Robin

Jasiulewicz-Kaczmarek, M.

Javed, Abbas

Javed, Arshad Ali

Javid, Roxana J.

Javierre-Lardies, Carlos

Jayasinghe, Shantha

Jeannotte, Sharon

Jenkins, Jesse

Jensen, Jesper Ole

Jensen, Per Anker

Jeon, Jeonghwan

Jeong, Bongju

Jeong, Suk-Jae

Jervis, Emily

Jewitt, Sarah

Ji, Jie

Ji, Qiang

Ji, Zhanlin

Jia, Fu

Jia, Junbo

Jia, Lili

Jia, Xinhua

Jiang, Bin

Jiang, Leiwen

Jiang, Shan

Jiang, Xiaoguang

Jiao, Hao

Jiao, Limin

Jiménez, Sofía

Jiménez-Ballesta, R.

Jin, Di

Jin, Xinfang

Jiricka, Alexandra

Jo, Ho Young

Johansson, Pär

John, Ranjeet

Johnes, Geraint

Johns, Raechel

Johnson, Glenn S.

Johnson, Matthew P.

Johnson, Mikael

Johnstone, Phil

Jokela, Päivi

Jokinen, Pekka

Jolly, Suyash

Jones, Benjamin

Jones, Calvin

Jones, Dwane

Jones, Julian
Jones, Kristal

Jones, Luke R.

Jones, Peter

Jones, Rebecca

Jones, Steven

Jonk, Yvonne

Jørgensen, Gertrud

Jorgenson, Jake D.

Joseph, Paul

Jou, Yung-Tsan

Jourdain, Damien

Jovanović, Marina

Juaidi, Adel

Juana, Luis

Jucker, Rolf

Julian, Kristi

Jun, Sunghae

Jung, Ha-Brookshire

Jung, Jaeyoung

Jung, Sojin

Jung, Timothy

Jungbluth, Niels

Junquera, Beatriz

Jurik, Nancy

Jusoh, Ahmad

Justo, Jackson John

Justo, Rachida

Jusuf, Steve Kardinal

Kabisch, Nadja

Kabitzsch, Klaus

Kachniewska, Magdalena

Kadiyala, Akhil

Kahil, Taher

Kaitna, Roland

Kalafatis, Scott

Kalantari, Zahra

Kalantari Soltanieh, Mohsen

Kaldellis, John K.

Kaller, Mike

Kalliopi, Droutsa

Kaltschmitt, Martin

Kalve, Shweta

Kamalahmadi, Masoud

Kamara, John

Kampas, Athanasios

Kamsu-Foguem, Bernard

Kanakoudis, Vasilis

Kanel, Sushil R.

Kang, Chaogui

Kang, He-Yau

Kang, Jungho

Kang, Myung Joo

Kang, Parminder Singh

Kang, Taeghyun
Kannan, Narayanan

Kansal, Monika

Kanwar, Pooja

Kao, Yung-Chou

Kaplan, Sigal

Kaplinsky, Raphael

Kaplon, Jan

Karamanos, Anastasios G.

Karan, Ebrahim P.

Karbuz, Sohbet

Karimi, Farrokh Jazizadeh

Karimi, Saeid

Karimpour, Mahsa

Karipidis, Philippos

Karlen, Doug

Karner, Alex

Karvonen, Andrew

Katayama, Shigeru

Katz-Rosene, Ryan

Katzschner, Lutz

Kaucic, Massimiliano

Kauffman, Robert J.

Kauko, Leiviskä

Kaup, Brent Z.

Kay, Mark J.

Kaynakli, Omer

Kazana, Vassiliki

$\mathrm{Ke}$, Jing

Keane, Robert E.

Kedron, Peter

Keels, Kay

Keil, Roger

Kelepertzis, Efstratios

Kellison, Timothy B.

Kelsey, R. Heath

Kendal, Dave

Kent, M.G.

Keplinger, Christoph

Keramitsoglou, Kiriaki M.

Keramydas, Christos

Kernecker, Maria

Kessler, Jeff

Kettley, Sarah

Khadka, C.

Khajuria, Anupam

Khakzad, Nima

Khalili, Nasrin R.

Khalsa, Sat Darshan

Khan, Ahmed S.

Khan, Mohammad Saud

Khan, Zaheer

Kharrazi, Ali

Khasreen, Monkiz

Khatib, Tamer 
Khodaei, Amin

Khodayar, Mohammad

Khubchandani, Jagdish

Kian, Rozita

Kieckhäfer, Karsten

Kim, Bowon

Kim, Doo-Rae

Kim, Euijune

Kim, Hyun

Kim, Inhi

Kim, Jae Ik

Kim, Jang-ho Jay

Kim, Jimin

Kim, Jin Ki

Kim, Junbeum

Kim, Jung Tae

Kim, Kwang Sik

Kim, Kye Hoon

Kim, Saehoon

Kim, Seong Dae

Kim, Sumin

Kim, SungSuk

Kim, Sungwon

Kim, Suyi

Kim, Tae Wan

Kim, Taebok

Kim, Younhee

Kim, Younjun

Kim, Yuseung

Kimbrough, Sue

Kindu, Mengistie

Kingsley, Jonathan

Kiniry, James

Kinnane, Oliver

Kinuthia, John

Kircher, Manfred

Kirchgässner, Gebhard

Kirkham, M. B.

Kirsch, Therese

Kirschstein, Thomas

Kiso, Takahiko

Kitissou, Marcel

Kitsios, Fotis

Kivits, Rob

Klitkou, Antje

Klöckner, Christian

Klumpp, Matthias

Knab, Sebastian F.

Kneafsey, Moya

Knemeyer, A. Michael

Knieling, Jörg

Kniffin, Kevin

Knizley, Alta

Knoshaug, Eric
Knuth, Daniela

Knutsson, Per

Koch, Florian

Kocmanová, Alena

Koda, Eugeniusz

Koech, Richard

Koelbel, Julian

Koffi, Demagna

Kogg, Beatrice

Kokko, Ari

Koller, Istvan

Kollias, Christos

Kolmes, Steven A.

Komeily, Ali

Komilis, Dimitrios P.

Komljenovic, Dragan

Komninos, Nicos

Kondo, Kayoko

Kondo, Yasushi

Kongar, Elif

Koo, Choongwan

Koo, Tay T.R.

Kooperman, Gabriel

Koppel, Kadri

Köppel, Johann

Korhonen, Ilkka

Korolija, Ivan

Korres, George

Korsgaard, Steffen

Kosal, Margaret

Kosmas, C.

Kosnik, Lea-Rachel

Kosoy, Nicolas

Koster, Auriane Magdalena

Kostov, Philip

Kotcon, James B.

Kotnik, Toni

Kotval, Zenia

Kougias, Ioannis

Kouretas, Dimitrios

Kousoulidou, Marina

Koutropoulos, Apostolos

Koutsias, Nikolaos

Koutsias, Nikos

Koutsogeorgou, Eleni

Koutsoudis, Anestis

Kovacs, Jason

Kovács, Gyöngyi

Kowalczyk, Tammy

Kowalski, Gregory

Kozlowski, Karl

Krakauer, Nir Y.

Kralj, Anna

Krange, Olve
Krarti, Moncef

Krasny, Marianne

Kravari, Kalliopi

Kreilkamp, Edgar

Krewenka, Kristin Marie

Krishnamurthy, P.K.

Krishnan, Sarada

Krometis, Leigh-Anne H.

Krull, Claudia

Krumay, Barbara

Kruse, Agneta

Kuang, Yaoqiu

Kubickova, Marketa

Kueneman, Eric

Kuik, Onno

Kulawardhana, Ranjani W.

Kumar, Manish

Kumar, Sandeep

Kumar, Subhash

Kumar, Surender

Kumbaroglu, Gürkan

Kung, Chih-Chun

Kuntke, Philipp

Kuntz, Marcel

Kunz, Reinhard

Kuo, Tsai-Chi

Kupecki, Jakub

Kurnia, Sherah

Kurniawan, Adi

Kurokura, Hisashi

Kurppa, Sirpa

Kusadokoro, Motoi

Kusch, Sigrid

Kuusela, Olli-Pekka

Kvarnström, Elisabeth

Kwiecień, Janusz

Kwon, Changhyun

Kwon, Hyun Joo

Kwon, Oh Kyoung

Kyriakarakos, George

Kyriazopoulos, Apostolos

La Notte, Alessandra

La Rocca, Rosa Anna

La Rosa, Daniele

La Scalia, Giada

Labeaga, José M.

Lacaze, Bernard

Lachowicz, Stefan

Lacombe, Donald J.

Lacomussi, Paola

Laes, Erik

Lafleur, Benoit

Lagabrielle, Erwann

Lagerqvist, Yayoi 
Lahr, Michael

Lai, Chi-Ming

Lai, Joseph, H.K.

Lai, Maria Bonaria

Lai, Sabrina

Lairon, Denis

Laitila, Thomas

Lajunen, Antti

Lam, Hon -Ming

Lam, Patrick T.i.

Lamastra, Lucrezia

Lambert, Thibaud

Lamers, Machiel

Lami, Isabella M.

Lan, Jiang

Landi, Daniele

Landman, Karen

Landry, Guylaine

Lanfranchi, Vincent

Lang, Chunmin

Langellotto-Rhodaback, Gail

Langergraber, Günter

Langhorst, Joern

Langlet, $\mathrm{T}$.

Langrell, Stephen R. H.

Langston, Craig

Lankford, Bruce

Lankoski, Leena

Lantieri, Claudio

Lantz, Frédéric

Lanzoni, Luca

Laperche, Anne

Lardies, Carlos Javierre

Larkin, Andrew

Larsen, Larissa

Larson, Paul D.

Lauren, Ari

Laureti, Tiziana

Lavoie, Roxane

Law, Rob

Lawhon, Mary

Lawrence, Judy

Lawrence, Theodore

Lawson, Emily C.

Lay, C.H.

Lay, Jann

Lazzari, Stefano

Le Heron, R.B.

Leach, Joanne

Lea-Cox, John

Leakey, Roger

Leal-Arcas, Rafael

Leccese, Franesco

Lechner, Alex
Lechtenböhmer, Stefan

Lee, Amy H. I.

Lee, Chien-Chiang

Lee, Christopher H. T.

Lee, Diane

Lee, Eunsu

Lee, Gene Moo

Lee, Hian Kee

Lee, Huen

Lee, In

Lee, Jae-Seung

Lee, Jen-Yao

Lee, Juhyun

Lee, Kun Chang

Lee, Kwan Ok

Lee, Maria R.

Lee, Min-Dong Paul

Lee, Sangbok

Lee, Sang-Geol

Lee, Sungjoo

Lee, Timothy

Lee, Tsung Hung

Lee, Uen-Do

Lee, Wai-ling

Lee, Yong-Gil

Lefticariu, Liliana

Lein, James

Leipner, Jörg

Leipold, Sina

Leitão, Nuno Carlos

Lemi, Adugna

Lennox, James

Lenzen, Manfred

León, Javier

Leonard, Rosemary Jill

Leonforte, Fabrizio

Leou, Rong-Ceng

Lessmann, Kai

Letizia, Paolo

Leung, David W. M.

Leung, Winnie S.C.

Leva, Maria Chiara

Levin, Noam

Lewandowski, Mateusz

Lewis, John

Li, Ainong

Li, Danny Hin Wa

Li, Dapeng

Li, Guang

Li, Han

Li, Hui

Li, Jian

Li, Jing

$\mathrm{Li}, \mathrm{Ke}$
Li, Peter Zhixiong

Li, Ping

Li, Qiang

Li, Rita Yi Man

Li, Ruopu

Li, Sen

Li, Simon

Li, Xianchang

Li, Xiaojun

Li, Xiwang

Li, Yangfan

Li, Yingzhu

Li, Yongliang

Li, Zhaohui

Li, Zhigang

Li, Zhixia

Li, Zukui

Liang, AN

Liang, Binqing

Liang, Fuyuan

Liang, Jianming

Liang, Jiaqi

Liang-Davidson, Tina

Liao, Haifeng

Liao, Kuei Hsien

Lichtenstein, Benyamin B.

Liddle, Brantley

Lie, Tek Tjing

Ligot, Gauthier

Lillo, Pau

Lim, Jae-Han

Lim, Michael K.

Lim, Steve C.

Lima, Joana

Lin, Brenda

Lin, Chin-Tsai

Lin, Hanzhi

Lin, Huilong

Lin, Jeng-wen

Lin, Shu-Jou

Lin, Yuan Hsu

Lindsey, Robin

Ling, Chris

Linke, Andrew

Linnér, Björn-Ola

Liou, Jiann-haw

Lioukas, Spyros

Lipinski, John

Lippke, Bruce

Little, Adrienne B.

Littlemore, James

Liu, Antung Anthony

Liu, Chao

Liu, Chunhua 


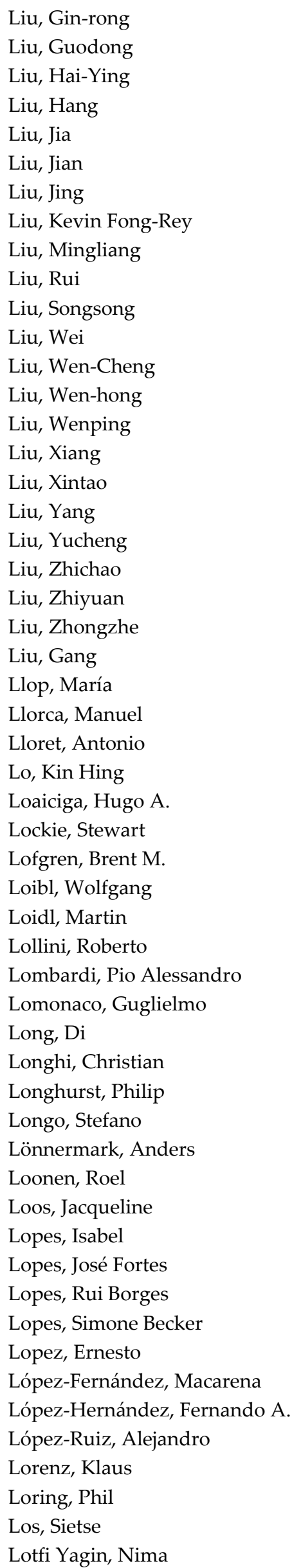

Lourenço-Gomes, Lina

Louwaars, Niels

Love, Jenny

Love, John

Low, Sui Pheng

Lowe, Christopher N.

Lowry, Gordon

Lozano, Rodrigo

Lozano-Oyola, Macarena

Lu, Chien-Yu

Lu, Jiaying

$\mathrm{Lu}$, Lin

Lu, Weizhen

Lu, Yonglong

Luc, Malgorzata

Lucas, Steven

Lue, Ko-Huang

Lugauer, Steven

Luis, Patricia

Lujano-Rojas, Juan M.

Luke, Christina

Lundhede, Thomas

Lung, Sidney

Luo, Jun

Luo, Zhongkui

Lupini, Antonio

Luxton-Reilly, Andrew

Lyng, Kari-Anne

Lyons, Padraig

Lyu, Zhe

M. Dewan, Ashraf

$\mathrm{Ma}$, Tan

$\mathrm{Ma}, \mathrm{Tao}$

Maalouf, Chadi

Mabit, Stefan Lindhard

MacAdam, Jennifer W.

Maccaferri, Simone

Maccarrone, Vincenzo

MacCarthy, Michael

MacDonald, Graham

MacGregor, Colin

Machado, Elia A.

Machairas, Vasileios

Macharis, Cathy

Mack, Elizabeth

Macken-Walsh, Áine

MacRae, Rod

Madaleno, Mara

Maddison, David

Magazzino, Cosimo

Magliocco, Adriano

Magnani, Natalia

Mahajan, Devinder

Mahdiloo, Mahdi
Maheshwari, Pankaj

Mahmoud, Moataz

Mahon, Marie

Maietta, Ornella Wanda

Mailhot, Mélina

Mair, Judith

Majewski, Janusz

Makatsoris, Harris

Málaga-Chuquitaype, Christian

Male, Frank

Malek, Ž.

Maletic, Matjaz

Malets, Olga

Malkinson, Dan

Mallee, Hein

Mallett, Oliver

Mañana-Canteli, Mario

Mancini, Lucia

Mancini, Marco

Mancini, Maria Cecilia

Mandilaras, Ioannis D.

Manes, Fausto

Manganelli, Benedetto

Mangano, Giulio

Mangold, Michael

Manner, Hans

Manning, Louise

Manso, José R. Pires

Mantas, Vasco.M.

Manuela Portela, Maria

Manzardo, Alessandro

Manzo, Stefano

Mao, Dehua

Marchi, Valentina De

Marcucci, Edoardo

Marcus, Alfred

Marfia, Gustavo

Margheritini, Lucia

Martinho, M.G.M.

Márialigeti, Károly

Marin, Giovanni

Markides, Christos

Marković, Todor

Marmiroli, Marta

Marone, Antonella

Marques, Maria Jose

Marques, Rui Cunha

Marra, Marianna

Marrone, Stefano

Marshall, Maria I.

Marshall, Nadine

Marsiglio, Simone

Marslev, Kristoffer

Marta-Costa, Ana Alexandra 
Marteau, Theresa M.

Martí Albiñana, José V.

Martí Albiñana, José Vicente

Marti-Ballester, Carmen-Pilar

Martin, Dominique Philippe

Martin, Michael

Martin, Wade E.

Martinet, Vincent

Martinez, Luis

Martinez, Manuel

Martinez-Alonso, Celia

Martínez-Ferrero, Jennifer

Martinho, V.J.P.D.

Martino, Gaetano

Martinopoulos, George

Martin-Rios, Carlos

Martín-Rubio, I.

Marucci, Alvaro

Maruyama, Takuya

Marx, Jerry

Marzaioli, Rossana

Marzano, Mariella

Marzano, Vittorio

Marzucchi, Alberto

Masala, Elena

Mase, Amber Saylor

Maserti, B.E.

Masiero, Mauro

Masoumi, Houshmand E.

Mas-Pla, Josep

Massaccesi, Luisa

Mastromarco, Camilla

Mastrucci, Alessio

Mas-Tur, Alicia

Masui, Keijiro

Mate, Vicente Agueda

Materia, V.C.

Mateus, Ricardo

Matharu, Avtar

Mathias, James

Matias, João C. O.

Matos, Cristina

Matous, Petr

Matschoss, Kaisa

Matsika, Emmanuel

Matsui, Kenji

Matsushita, Yoshiki

Matthews, Alan

Matthews, Stephen N.

Mattoni, Benedetta

Matzler, Kurt

Mauerhofer, Volker

Mauro, Gerardo Maria

Mauro, Giovanni
Mavropoulos, Georgios

Maw, Michael J.W.

Mayer, Audrey

Mayer, Helmut

Maynard, Leigh

Mayo, Glenda

Mazaheri, Mandana

Mazhar, Ilyas Mazhar

Mazumder, Malay

Mazzocchi, Chiara

Mazzulla, Gabriella

McAreavey, Ruth

McArthur, David

McAssey, Edward

Mccabe, Scott

McCann, Philip

McCartney, Shelagh

McCarty, Jessica

McCauley, Darren

McConkie, Eileen

McCord, Michael

McCrackin, Michelle

Mcdonald, Scott

McDonald, Yolanda Jane

Mcewan, Ryan

McGarry, Scott

McGinnis, Michael

McIntosh, James

McLaren, Joyce

McLellan, Benjamin Craig

McLeman, Robert

McLeod, Fraser

McMeeking, Kevin

McNabola, Aonghus

McNeil, Jacqueline

McPhillips, Lauren

McQuaid, Ronald W.

Medeiros, Eduardo

Medina, N. Flores

Meegoda, Jay N.

Meek, Jack W.

Meerman, Hans

Meerow, Sara

Meethan, Kevin

Meglio, Olimpia

Mehan, Sushant

Mehring, Marion

Mei, Ciqi

Meier, Fred

Meier, $\mathrm{H}$.

Meier, Helena

Meijers, Evert

Meisenberg, Gerhard

Mekonnen, Mesfin Mergia
Melendez-Pastor, Ignacio

Melis, Roberta

Melland, Alice

Melo, Patricia

Meloni, Italo

Memary, Reza

Memon, Fayyaz Ali

Memon, Muhammad Saad

Menaa, Farid

Mendelsohn, Robert O.

mendiola, José

Menegaki, Angeliki

Menendez, Hector

Menezes, Moisés Lima De

Meng, Bo

Meng, Jinghui

Meng, Lexuan

Meng, Sam

Meng, Xiangning

Meng, Xiaoliang

Menoufi, Karim

Mentis, Dimitris

Meo, Mark

Mereu, Valentina

Merli, Roberto

Merritt, Eileen

Mertes, Carly M.

Messina, Fabrizio

Messner, Jakob W.

Meyer, Claas

Meyer, Denny

Meyers, Jacqui

Mguni, Patience

Mi, Zhifu

Michael-Tsabari, Nava

Michailides, Constantine

Michailidis, Anastasios Ch.

Migliore, Giuseppina

Mihet-Popa, Lucian

Milani, Dia

Milenkovic, Milos

Militino, Ana Fernández

Miliute-Plepiene, Jurate

Millar, Michelle

Miller, Gloria

Miller, Robert A.

Miller, Sini

Miller, Wendy

Miller, William M.

Mills, Morena

Min Aung, Myo

Minakata, Daisuke

Minciullo, Marco

Minguillón, María Cruz 
Minx, Jan C.

Miras, Pedro Zorrilla

Miret-Pastor, Lluís

Mirkouei, Amin

Mirosa, Miranda

Mirvis, Philip

Mirza, Babur

Mirzabaev, Alisher

Mishra, Aditya K.

Mishra, Ashok

Mishra, Asit Kumar

Mishra, Gouri Shankar

Mishra, Phoolendra

Mishra, Sabyasachee

Mitchell, Piers D.

Mitra, Chandana

Mittal, Nidhi

Miyamoto, Hitoshi

Mizgier, Kamil

Mlsna, Todd

Mo, Jinhan

Modaresi, Roja

Moeller, Carina

Moggi, Sara

Moghanloo, R.G.

Moglia, Magnus

Mohajeri, Nahid

Mohammadi, Amir H.

Mohammadian, Masoud

Mohlakoana, Nthabi

Mohtar, Rabi H.

Moldan, Bedrich

Moldavanova, Alisa V.

Molderez, Ingrid

Moles, Richard

Molina Gonzalez, Jose Luis

Molina-Navarro, Eugenio

Möller, Greg

Møller-Jensen, Lasse

Molowny Horas, Roberto

Mondragon, R. J.

Monforti-Ferrario, Fabio

Moniruzzaman, Md

Montazeri, Hamid

Montllor, Joan

Monzon, Andrés

Moobela, Cletus

Moon, Christopher J.

Moon, Zola Knowles

Moons, Ingrid

Moore, Nathan

Moore, Philip

Moos, Markus

Mora, Cristina
Mora, Rodrigo

Morales, L. Emilio

Moran, Brooke

More, Elizabeth

Moreira, Francisco

Moreno Chou, Ana Tsui

Moretto, Antonella

Morgenstern, Paula

Mori, Koichiro

Moriarty, Patrick

Morioka, Yushi

Moriondo, Marco

Morley, Bruce

Morley, Bruce

Morone, Piergiuseppe

Morrison-Saunders, Angus

Morrissey, Anne

Morrissey, Karyn

Mosadeghi, Razieh

Moscordo, Gianna

Moscou, Matthew

Moss, Todd

Moss, Todd W.

Motallebi, Marzieh

Mottola, Fabio

Motuzienè, Violeta

Mousavi, Seyedesmaeil

Moutinho, Victor

Moutopoulos, Dimitrios K.

Mowry, Greg

Mozumder, Pallab

Mueller-Warrant, George W.

Mugera, Amin W.

Muis, Sanne

Mukherjee, Zinnia

Mukhopadhyay, Jaya

Mukul, Sharif

Müller, Daniel

Müller, Felix Claus

Müller, Jochen A.

Müller, Johannes

Müller, Matthias

Mulligan, Kate

Mulvihill, Peter R.

Mummolo, Giovanni

Muneepeerakul, Rachata

Munoz, Macarena

Münter, Angelika

Mur Lacambra, Jesús

Muraca, Barbara

Murano, Peter

Murata, Akinobu

Murgante, Beniamino

Murphy, Fiona
Murtagh, Niamh

Murungweni, Chrispen

Musco, Francesco

Musso, Fabio

Muttarak, Raya

Mwakalonge, Judith L.

Myeong, Seunghwan

Myers, James

$\mathrm{Na}$, Byungsoo

Nackoney, Janet

Naegele, Hans-joachim

Nahorski, Zbigniew

Najm, Husam

Nakamura, Masao

Nakandala, Dilupa

Nakano, Katsuyuki

Namiesnik, Jacek

Nandwani, Dilip

Nanos, Nikos

Napoli, Christian

Napoli, Grazia

Napp, Tamaryn

Narayan, Seema

Nasahara, Kenlo

Naser, Hanan

Nasidi, Muhammad

Nässén, Jonas

Nastasi, Benedetto

Nathan, Iben

Nathan, Maria

Naugle, Asmeret Bier

Naumann, Stefan

Nayak, Dali

Nchanji, Eileen Bogweh

Neave, Melissa

Neculita, Carmen Mihaela

Nedelko, Zlatko

Nehren, Udo

Nelson, Michael A.

Nelson, Tim

Nemetz, Peter N.

Nepveu, M.

Neugebauer, Sabrina

Neuts, Bart

Nevens, Frank

Neves, Orquídia

Neves, Rui

New, Steve

Newman, Erica A.

Newman, Galen

Newman, Lenore

Newman, Peter

$\mathrm{Ng}$, ChoNam

$\mathrm{Ng}$, Derrick Wing Kwan 
Ng, Kelvin T. W.

$\mathrm{Ng}$, Lisa C.

Nghiem, Son

Ngoy, Kikombo

Nguyen, T.Thanh-Binh

Nicholls, Sarah

Nichols, Peter

Nicolau, Juan Luis

Nicolli, Francesco

Niedziałkowski, Krzysztof

Nielsen, Jonas Østergaard

Nielsen, Per Sieverts

Niero, Monia

Nigim, Khaled

Nigro, Marialisa

Niimi, Yoko

Niimimaki, Kirsi

Nijland, Wiebe

Nikitas, Alexandros

Nikolaeva, Ralitza

Nikolaou, Ioannis

Nikolas Müller, Architekt

Nilsson, Isabelle M.

Nilsson, R. Henrik

Niroumand, Amir M.

Nishioka, Shuichiro

Nissi, Eugenia

Nitti, Michele

Niu, Jianlei

Niven, Robert

Nixon, Hilary

Nobile, Lucio

Nocera, Francesco

Nomura, M.

Norici, Alessandra

Norman, Rosemary

Noro, Marco

Noronha, Carlos

Norouzzadeh, Payam

Norrman, Jenny

Noshadravan, Arash

Notarnicola, Bruno

Noubactep, Chicgoua

Nour, Mutasim

Novas, Nuria

Novelli, Marina

Noyons, Ed

Nubani, Linda

Nukpezah, Julius

Nunan, Fiona

Nunes, Sandra

Nunes-Vaz, Rick

Nuss, Christian

Nyangweso, Mary
O'Hara, Sarah

Oates, Caroline J.

Obonyo, Esther A.

O'Boyle, Louise

O’Brien, Danny

Ochatt, Sergio J.

Ochuodho, Thomas O.

O'Connor, Justin

Oechsner, Hans

Oelberman, Maren

Oenema, Oene

Ogutu, Joseph O.

Oh, Dong-hyun

O'Hagan, Anne Marie

O'Hara, Kevin

Ohno, Hajime

Ojeda Benitez, Sara

Ojovan, Michael

Okafor, Florence

Ólafsdóttir, Guðrún

Olander, Stefan

Oliveira, Teresa

Oliver, Matthew

Olofinjana, Ayodele

Olson, David L.

Olson, Larry

Omar, Ayman

Omar, Mohammed A.

Omar, Noshin

Omitaomu, Olufemi A.

O'Neill, Kirstie

Ong, Ghim Ping Raymond

Ongondo, Francis Otieno

Onkila, Tiina

Onyango, Vincent

Oort, Niels Van

Opp, Susan M.

Orbach, Raymond L.

O'Rear, Eric G.

Orford, Scott

Orlov, Anton

Orosa, José

Orsi, Francesco

Ortega, Manuel Alcazar

Ortiz, F.J.G.

Ortiz, Rodomiro

Ortiz-Rodríguez, David

Osmond, Paul

Ostermeyer, York

Ouenniche, Jamal

Oueslati, Walid

Owen, Luke

Ozawa-Meida, Leticia

Özerol, Gül
Ozguven, Eren Erman

Paatero, Jukka

Pabel, Anja

Pablo-Romero, María Del P.

Pace, Stefano

Pacheco Torgal, F.

Pacheco-Blanco, B.

Pacheco-Torres, R.

Pachego-Torgal, F.

Paddison, Ronan

Padeiro, Miguel

Paderni, Paola

Padin, Carmen

Padovan, Dario

Padovani, Emanuele

Padowski, Julie

Paeleman, Ine

Pagliaro, Mario

Pal, Anamitra

Pal, Pinaki

Pal, S.

Palazzo, Danilo

Palcic, Donal

Palella, Boris Igor

Palitzsch, Wolfram

Palm, Jenny

Palma, Marco A.

Palmer, Graham

Panagiotaras, Dionisios

Panagiotidis, Theodoros

Panagiotis, Elias

Panagopoulos, Thomas

Pande, Saket

Pandey, Bhartendu

Pandit, Arka

Panepinto, Deborah

Paneque, Pilar

Pang, Shusheng

Pang, Xiufeng

Pangbourne, Kate

Pant, Deepak

Pantaleo, Antonio Marco

Pantera, Anastasia

Panuwatwanich, Kriengsak

Panwar, Rajat

Panzone, Luca A.

Papa, Matteo

Papachristos, Georgios

Papanikolaou, Ioannis

Papargyropoulou, Effie

Papathanasiou, Natalia

Pappalardo, Giovanna

Papurello, Davide

Parajuli, Rajan 
Paravantis, John A.

Parcero-Oubiña, César

Pardo-del-Val, Manuela

Parece, Tammy E.

Paredes, Javier

Paredes, Paula

Parisi, Domenico

Park, Andrew

Park, Eunil

Park, Hung-suck

Park, Jae

Park, Kun Soo

Park, Sang-Chul

Park, Sehyun

Park, You-Jin

Parkany, A. Emily

Parker, Nathan

Parkins, John R.

Parkinson, Jonathan

Parmentier, Benoit

Parsa, H.G.

Parsons, Chris

Partidario, Maria Rosario

Pascucci, Stefano

Pasek, Zbignew

Pasman, Hans J.

Passarella, Giuseppe

Pastor, Maria Calero

Pätäri, Satu

Patchigolla, Kumar

Pate, Michael

Pateman, Hilary

Paterakis, Nikolaos

Pathan, Al Sakib Khan

Patton, Allison P.

Paul, Debdeep

Paul, Nicholas

Paül, Valerià

Paulsen, Hans Marten

Paulsson, Jenny

Pawar, Kulwant

Paz-Ferreiro, Jorge

Pearce, John A.

Pearce, Joshua M.

Peck, David

Pecori, Riccardo

Pegallapati, A.K.

Pekkanen, Petra

Peldschus, Friedel

Pelkey, Neil

Pelletier, Vincent

Pellitero, Ramón

Pellizzoni, Luigi

Peña-García, A.
Peñasco, Cristina

Peng, Yi

Penz, Elfriede

Pepe, Antonio

Pepe, Marco

Perboli, Guido

Perdiguero, Jordi

Perea Moreno, Alberto

Pereira, Amaro

Pereira, Angela

Pereira, Dolores

Pereira, Luis S.

Pereira, Paulo

Perera, Kithsiri

Perez, Francesca

Pérez, Rocío

Pérez-Badia, Rosa

Perez-Collazo, Carlos

Pérez-Gladish, Blanca

Pérez-López, Raúl

Perkol-Finkel, Shimrit

Perković, Luka

Perry, Chris

Perry, Jim

Perry, Patsy

Persson, Åsa

Peruzzini, Margherita

Pesch, Udo

Peterkova, Katerina

Petković, Dalibor

Petroselli, Andrea

Petrossian, Gohar A.

Petrykowski, Piotr

Pettenella, Davide

Petti, Luigia

Pezzotta, Giuditta

Philipp, Conrad

Phillips, Gavin

Phillips, Lynn Roche

Phipps, David J.

Piccini, Chiara

Pichler, Paul

Pickett, Steward

Pienaar, Josua

Pierson, Kawika

Pietrelli, Loris

Pietsch, Marilyn

Pillay, Renginee

Pilloni, Virginia

Pimm, Stuart L.

Pinar Pérez, Jesús María

Pincetl, Stephanie

Pinho, Carlos

Piniella, Francisco
Pinkerton, Kent

Pinto, Hugo

Pinto, Rute

Pinto-Correia, Teresa

Pinto-Varela, Tânia

Piot-Lepetit, Isabelle

Pires, J.C.M.

Piribauer, Philipp

Piscart, Christophe

Pisello, Anna Laura

Pistolesi, Marco

Plakas, Konstantinos

Plaza, Beatriz

Pliakos, Konstantinos

Plumecocq, Gael

Plutzar, Christoph

Poerschke, Ute

Poirier, Alexandre

Pojani, Dorina

Pokrajac, David

Polido, Alexandra

Polinori, Paolo

Polk, Merrit

Pollack, Keshia

Pollitt, Hector

Pollitt, Michael

Pols, A. J. K.

Polzin, Steven

Pons, N.A.D.

Pons, Oriol

Ponte, Stefano

Ponzoni, Flavio

Pookulangara, S.

Popp, József

Pore, Madhurima

Portner, Brigitte

Portney, Kent E.

Posa, Antonio

Poulikidou, Sofia

Powe, Neil

Powell, Kody M.

Powell, Lisa

Powers, Matthew

Prada, Alessandro

Pradhan, Prajal

Prasad, Monica

Prescott, John E.

Presno Casquero, M.J.

Press, Melea

Prester, Jasna

Pretlove, Stephen

Prezioso, Giuseppina

Pribadi, Didit Okta

Prideaux, Bruce 
Prieto, Miguel

Prihodko, Lara

Pritchard, Bill

Privitera, Donatella

Prochnow, Annette

Proenca, Sara

Proietti, Stefania

Prosperi, Maurizio

Przychodzen, Wojciech

Pucker, Johanna

Pueyo, Ana

Pueyo Anchuela, Ó.

Pugh, Thomas

Puig, Martì

Puliafito, Antonio

Pulido-Fernández, Manuel

Pulugurtha, Srinivas S.

Purkus, Alexandra

Pushkar, Svetlana

Putrino, David

Puumalainen, Kaisu

Pype, Marie-Laure

Pyrialakou, V. Dimitra

Qaim, Matin

Qattawi, Ala

Qian, Qin

Qian, Zhu

Qiao, Lei

Qin, Yuanwei

Qing, Lu

Qiu, Jiangxiao

Qiu, Yueming

Qiu, Zeyuan

Qu, Shen

Qudrat-Ullah, H.

Quental, Nuno

Quieti, M.G.

Quintela, J.A.

Ra, Changsix

Raa, Birger

Raab, Carola

Raboni, Massimo

Rada, Elena

Rada, Elena

Radcliffe, Jonathan

Radfar, M.

Rafiq, S.

Rahman, Munshi

Rahnamayiezekavat, P.

Rajkovich, Nicholas

Ralf, Risser

Del RíoRama, M.C.

Ramamurti, Ravi

Ramani, Raja
Ramezani, Mohsen

Ramezanpour, Ahad

Ramirez, Alvaro

Ramirez, Bernardo

Rammer, Werner

Ramos, Nuno

Ramos Miras, J.J.

Ramos Soto, Alejandro

Ramos-Real, F.J.

Ramseook-Munhurrun, P.

Rana, Padmakshi

Ranchhod, Ashok

Ranieri, Ezio

Rao, Bharat

Rao, P.N.

Räsänen, Aleksi

Rashid, Amir

Rassaei, Farshad

Rau, Henrike

Rauch, Erwin

Raugei, Marco

Rauland, Vanessa

Rauter, Romana

Ravazzoli, Elisa

Ravn, Søren Hove

Raymond, Leigh S.

Raynham, Peter

Razmerita, Liana

Razul, Azad

Rebennack, Steffen

Reboredo, Juan C.

Recker, Jan

Redsteer, Margaret Hiza

Reed, Dorothy A.

Rees, Simon

Reeve, Jennifer

Rega, Carlo

Reguero, Borja

Reid, Doug

Reilly, John

Reimer, Adam

Reino, Luís

Reis, Arianne C.

Reisi, Marzieh

Reiss, Kelly Chinners

Reisslein, Martin

Remmel, Tarmo

Ren, Hao

Ren, JingZheng

Rendleman, C. Matthew

Renna, Paolo

Renné, David S.

Repka, Sari

Rese, Alexandra
Resop, Jonathan

Reuter, Ryan

Revoredo-Giha, Cesar

Reyes, José Santos

Reyes-Rodríguez, J.F.

Rezg, Nidhal

Rhee, Seung-Yoon

Rhiney, Kevon C.

Rhodes, Catherine

Ricart Casadevall, S.

Ricciardi, Paola

Rice, James B.

Richardson, James

Rider, Traci Rose

Ridley, Luke

Riel, Andreas

Riemer, Manuel

Rienow, Andreas

Riggio, Heidi R.

Righini, Gaia

Rinaldi, Fabio

Rinaldi, Stefano

Ringhofer, Lisa

Ringwood, John

Riopel, Diane

Rioux, Liliane

Ristimäki, Miro

Ritzén, Sofia

Ritzoulis, Christos

Rivas-Casado, Monica

Rivera, Francisco F.

Rivest, David

Rizio, Dina

Robbins, Derek

Roberts, Dar

Robinson, John

Robledo, Carmenza

Robotis, Andreas

Roca, Lidia

Rocchi, Lucia

Rocha, A.

Rocha, Juan-Carlos

Rode, Johannes

Rodela, Romina

Roderick, Hugh

Roders, Ana Pereira

Rodil, Óscar

Rodrigues, Marcos

Rodrigues, Marta

Rodriguez, Luiz Carlos E.

Rodríguez-Antón, J.M.

Rodríguez-Cohard, J.C.M.

Rodríguez-Couto, S.

Rodriguez-Garcia, G. 
Rodríguez-Labajos, B.

Rodríguez-Soalleiro, R.

Rodziewicz, Joanna

Roebeling, Peter

Roehrich, Jens

Roessler, Regina

Rogers, David

Rogers, John G.

Rogers, Judy

Roh, Taewoo

Roig-Tierno, Norat

Rojas, Carolina

Rollings, Kim

Rollins, Rick

Rollins, Rick

Romagnoni, Piercarlo

Román, Rocio

Romano, Bernardino

Romero, Inmaculada

Romero-Troncoso, Rene

Rommel, Jens

Roorda, Matthew J.

Rosa, Isabel

Rosati, Luca

Rosburg, Alicia

Rösch, Christine

Roscia, Mariacristina

Rose, Geoffrey

Rosehart, William

Rosenkranz, Andreas

Roset, Jaime

Rosewarne, Stuart

Ros-McDonnell, Lorenzo

Roson, Roberto

Rössel, Jörg

Rossi, Federica

Rossi, Giuseppe

Rota, Maria

Roth, Guy

Roumpos, Christos

Rousseau, Sandra

Rowe, James

Roy, Jayjit

Rozakis, Stelios

Ruark, Matt

Ruddell, Darren

Ruffino, Barbara

Rufin, Carlos

Ruggiero, John

Ruiz, Cristián Ducoing

Ruiz-Gallardo, José Reyes

Rulli, Maria Cristina

Rupprecht, Christoph D.D.

Rus, Rozalia Veronica
Russak, Amos

Russo, Ivan

Rusu, Eugen

Rutt, Rebecca Leigh

Ryan, Geraldine

Ryan, Jean

Ryan, Susan

Rydzek, Benedikt

Rye, Johan Fredrik

Ryu, Doojin

Saarinen, Jarkko

Sabaghi, Mahdi

Sabio, Nagore

Sacande, Moctar

Sacco, Pierluigi

Sack, Detlef

Sadatsafavi, Hessam

Šadauskienė, Jolanta

Sadd, Debbie

Saddler, Hugh

Sadler, Richard Casey

Saelens, Dirk

Safacas, Athanasios

Safdarnejad, Seyed Mostafa

Safiuddin, Md.

Sage, Colin

Sági, László

Saha, Subrata

Sahoo, Alok K.

Sahota, Parminder

Sajjadian, Masoud

Sala, Serenella

Salanova Grau, Josep Maria

Salas, Vicente

Salata, Ferdinando

Salcedo Sanz, Sancho

Salerno, Daniela

Salerno, Franco

Salinas, Carla

Sallak, Mohamed

Sallam, Mohamed

Salmon, Christian

Salmon, John

Salomone, Roberta

Salvalai, Graziano

Salvia, Rosanna

Salvioni, Daniela M.

Salvo, Francesca

Samadani, Ehsan

Sample, David

Sanchez, Benito

Sánchez, María

Sánchez De La Flor, F.J.

Sánchez-Cañizares, S.M.
Sánchez-Lozano, J.M.

Sánchez-Medina, A.J.

Sánchez-Moral, Simón

Sandell, Rickard

Sandholz, Simone

Sandström, Ulf

Sandwell, Philip

Sanesi, Giovanni

San-Jose, Leire

Sankey, Joel

Santafe, Miguel Redón

Santamaria, J.

Santamouris, Matheos

Santeramo, Fabio Gaetano

Santillan, Arturo Orozco

Santolini, Riccardo

Santos, Eduardo R.

Santos, José Maria

Santos, Maria Teresa L. Dos

Santos, Mónica

Santos, Paulo

Santoso, Surya

Sanyé-Mengual, Esther

Sapena, Juan

Saponara, Sergio

Sappa, Giuseppe

Saqib, Zafeer

Sarfaty, Mona

Sargolini, Massimo

Sarkar, Biswajit

Sarkar, Dripta

Sarnoski, Paul

Sartori, Martina

Sartre, Xavier Arnauld De

Sarukkalige, P.R.

Sasatani, Daisuke

Sassi, Paola

Saunders, Manu

Sautier, Marion

Savary, Serge

Säynäjoki, Antti

Scaglione, Giuseppe

Scalici, Massimiliano

Scargiali, F.

Scarpellini, Sabina

Schaefer, Anja

Schaefer, Klaus

Schaffartzik, Anke

Schaufele, Brandon

Scheinberg, Anne

Schelhas, John

Schenone, Corrado

Schepaschenko, Dmitry

Schepelmann, Philipp 
Scherer, Laura

Scherr, Sara

Scherrer, Maike

Schewenius, Maria

Schiemann, Frank

Schillings, Claudia

Schindler, Stefan

Schlange, Lutz E.

Schlesinger, William

Schlummer, Martin

Schmidt, Hans-Peter

Schmidt, Jörn O.

Schmidt, Mario

Schmidt, Rainer

Schmidt-Thomé, Philipp

Schmidt-Vogt, Dietrich

Schmutz, Vaughn

Schnabel, Marc Aurel

Schneibel, Anne

Schneidemesser, Erika Von

Schneider, Mindi

Schneider, Petra

Scholte, Jan Aart

Scholz, Johannes

Scholz, Roland

Schoon, Michael

Schoormans, J.P.L.

Schouten, Jaap

Schøyen, Halvor

Schröder, Dietrich

Schroth, Olaf

Schuck, Amie

Schulte, Bruce

Schulz, Jennifer J.

Schulze, Mike

Schumacher, Jens

Schüttrumpf, Holger

Schuurman, Dimitri

Schweinsberg, Stephen

Schweizer, Pia-Johanna

Schwer, Avery

Schwitzguebel, Jean-Paul

Sciortino, M.

Scioscia, Floriano

Sciurpi, Fabio

Scofield, John H.

Scott, James W.

Scott, Lloyd

Scott, Marian

Scotti, Nunzia

Scott-Parker, Bridie

Scozzafava, Gabriele

Scungio, Mauro

Sebastian, Leocadio
Secondi, Luca

Sedlacek, Sabine

Sedziwy, Adam

Seers, P.

Segatori, Raoul

Segoni, Samuele

Seidl, Roman

Seidler, Reinmar

Sekiguchi, Tomoki

Semanjski, Ivana

Semeraro, Teodoro

Senigaglia, Valeria

Senwo, Zachary

Seo, Youngmin

Serôdio, Carlos

Serrano, Tarik

Serrano González, Javier

Serrano-Cinca, Carlos

Serrano-Lujan, Lucía

Serrão, José Eduardo

Serrette, Reynaud

Sert, Sedef

Settanni, Ettore

Setzer, William

Severo, Marta

Seya, Hajime

Sgroi, Filippo

Shabani, Amir

Shackman, Joshua D.

Shah, Kalim U.

Shahabfar, Alireza

Shaheen, Susan

Shahid, Shamsuddin

Shahnia, Farhad

Shalaby, Ahlam I.

Shan, Yongwei

Shandra, John

Shane, David Grahame

Shang, Jin

Shang, Songhao

Shannon, Kelly

Shao, Changliang

Shao, Chaofeng

Shao, Guofan

Shao, Shuai

Sharif, Amir M.

Sharma, Amit

Sharma, Ashish

Sharma, Lakesh

Sharp, John M.

Sharpe, Tim

Shaw, Pete

Shealy, Tripp

Shearer, Christine
Sheehan, Kim

Shen, Junyi

Shen, Yung-Chi

Shepherd, Deborah M.

Sher, Willy

Sherchan, Samendra

Shi, Linda

Shi, Peijun

Shi, Suan

Shi, Wenbo

Shi, Xianming

Shi, Xiaoqing

Shi, Xunpeng

Shiau, Jenq-Tzong

Shiau, Tzay-An

Shields, Jeff

Shih, Hsu-Shih

Shim, Sang-Oh

Shimada, Hideki

Shimada, Koji

Shin, ChangHwan

Shin, Juneseuk

Shin, Jungyeop

Shishegar, Nastaran

Shogren, Jason Fredrick

Shotton, Elizabeth

Shoyama, Kikuko

Shrestha, Pramen

Shrivastava, Aatmesh

Shu, Chengli

Shu, Kesheng

Shu, Xiang

Shuai, Jia-Jane

Shyr, Oliver F.

Sidali, Katia Laura

Siebeneck, Laura

Siedentop, Stefan

Sieffert, Yannick

Sierocinski, Pawel

Sierra, Joan Pau

Siew, Renard Yung Jhien

Sigler, Thomas

Sika, Gbegbelegbe

Sillero, Neftali

Silva, Emmanuel Sirimal

Silva, Joaquim

Silva, Lígia

Silva-Send, Nilmini

Sim, Seung-Gyu (Andrew)

Simani, Silvio

Simioni, Michel

Simmons, Bora

Simões, Pedro

Simon-Rojo, M. 
Sims, Ronald C.

Sinclair, M.A.

Singh, Nirvikar

Singh, Simron Jit

Singh, Vivek

Sinnott, Derek

Sipponen, Mika Henrikki

Sisto, Roberta

Sisworahardjo, Nurhidajat

Sitko, Nicholas J.

Sitzia, Tommaso

Škec, Stanko

Skeffington, Richard

Skelhorn, Cynthia

Skierucha, Wojciech M.

Skilodimou, Hariklia D.

Skjelvåg, Arne Oddvar

Skoulou, Vassiliki K.

Skouloudis, Andreas N.

Skouri, Konstantina

Skutsch, Margaret

Slaev, Alexander

Sleight, Weldon

Slotkin, Michael H.

Small, Michael

Smeets, Paul

Smetschka, Barbara

Smirnova, Janna

Smith, Brianne

Smith, Geoff

Smith, Hollie

Smith, Kendra

Smith, Liam

Smith, Mark Stafford

Smith, Michael E.

Smith, Rod

Smith, Stephen L.J.

Smyth, Stuart J.

Snyder, Eric

Snyder, Thomas

Soda, Satoshi

Sodhi, ManMohan

Sohaib, Osama

Sohl, Terry

Sohn, Christophe

Sohn, So Young

Søholt, Susanne

Solaimani, Sam

Solís-Guzmán, Jaime

Solomon, Barry

Someth, Paradis

Song, Dong-Ping

Song, Doosam

Song, Li
Song, Manlin

Song, Shunfeng

Song, Suyong

Song, Wei

Sonnenschein, Michael

Sonter, L.J.

Sophocleous, Marios

Soppe, Birthe

Soriano, Domingo Ribeiro

Soscia, Isabella

Sotarauta, Markku

Sotirov, Metodi

Soto, Ignacio

Soto, Manuel

Soto Fernandez, David

Soukopová, Jana

Sousa, Luis Ribeiro E.

Sousa Fragoso, Rui

Soutar, Geoffrey N.

Southall, Ryan

Souza-Monteiro, Diogo M.

Spada, Matteo

Spak, Scott

Sparber, Wolfram

Sparrow, Sarah

Spatari, Sabrina

Speelman, E.N.

Spijker, Jeroen

Spilanis, Ioannis

Spinoni, Jonathan

Spinosa, Ludovico

Spit, Tejo

Srebotnjak, Tanja

Srinivasan, Mahesh

Srinivasan, Ravi S.

Srubar, Wil V.

Stadler, Konstantin

Stafoggia, Massimo

Ståhbröst, Anna

Stakhanov, Oleg

Stål, Herman

Stål, Herman I.

Stamatiou, Yannis C.

Stamatis, Nikolaos

Stanley, John K.

Stark, Sari

Staš, David

Stathis, Dimitrios

Stauvermann, P.J.

Stavi, Ilan

Stead, David

Steeman, Marijke

Stefani, Gianluca

Stefanidis, Kostas
Steinberger, Julia

Steiner, Daniel

Steiner, Frederick

Steinkellner, Siegrid

Stenvall, Jari

Stephan, André

Stephenson, Max O.

Šter, Branko

Stevenson, Kathryn

Stewart, Chris

Stewart, Iain

Stewart, James

Stindt, Dennis

Stockdale, Elizabeth

Stoep, Gail Vander

Stoett, Peter

Stöger, Eva

Stokes, David L.

Stoorvogel, Jetse

Stoppato, Anna

Stoutenborough, J.W.

Straub, Ad

Straub, Jeremy

Strazza, C.

Streblow, Rita

Strezov, Vladimir

Strohbach, Michael

Strubelt, Henning

Strumsky, Deborah

Strunz, Sebastian

Stubblefield, Andrew

Sturiale, Luisa

Sturm, Arnon

$\mathrm{Su}$, Bin

$\mathrm{Su}$, Ruoyu

Su, Shiliang

$\mathrm{Su}$, Tai-Sheng

$\mathrm{Su}$, Weizhong

Su, Ying-Ming

$\mathrm{Su}$, Yuan-Fong

Suarez, Rafael

Subramanian, Venkat

Suchenwirth, Leonhard

Sudbury-Riley, Lynn

Suen, I-Shian

Sugawara, Hirofumi

Suh, Dong Hee

Suhardiman, Diana

Sui, Sui

Suk, Sung Joon

Suk, Sunhee

Suleiman, Muhannad T.

Sullivan, Annett

Sumaili, Jean 
Summers, James Kevin

Sun, Caizhi

Sun, Daniel Jian

Sun, Jian

Sun, Laixiang

Sun, Xin

Sun, Ya-Yen

Sun, Zhanli

Sunam, Ramesh

Sunderer, Georg

Sundström, Aksel

Suntio, Teuvo

Suresh, Nallan C.

Surminski, Swenja

Sutherland, Fred

Sutton, Paul

Suuronen, Petri

Swinkels, Laurens

Syed, Ali Murad

Sylwester, Kevin

Syri, Sanna

Szabo, Sylvia

Szabó, Sandor

Szabó, Szilárd

Szekely, Gyorgy

Taboada, F.G.

Tachibana, Towa

Tada, Tsuyoshi

Tagliabue, Lavinia C.

Taheri, Javid

Tajeddini, Kayhan

Tajiri, Kazuya

Taka, Maija

Takahashi, Yoshi

Takao, Manabu

Takeuchi, Kenji

Tal, Alon

Tallon, Andrew

Tambo, Torben

Tamburrano, P.

Tamburrano, Paolo

Tamošaitienè, Jolanta

Tanaka, Nobuhiko

Tanaka, Shinsuke

Tanev, Stoyan

Tang, Huajun

Tang, Lina

Tang, Yu-Ting

Taniguchi, Eiichi

Tanim, Tanvir R.

Tapete, Deodato

Tappia, Elena

Tarantino, Eufemia

Tarazona Vento, A.
Tarí, Juan J.

Tartakovsky, L.

Tatano, Fabio

Tavanti, Marco

Tavor, Dorith

Taylor, Christopher

Taylor, Eric

Taylor, Tim

Tayyebi, Amin

Tchangani, Ayeley P.

Tchetchik, A.

Teh, Lydia

Tehrani, Fariborz M.

Tehrani, Rouzbeh

Tei, Alessio

Teixeira, Carlos Afonso

Teixeira, Ricardo F.M.

Teixeira, Samantha

Temple, Norman J.

Templeton, Michael

Ten Bhömer, Martijn

Tepe, Emre

Terlouw, Kees

Terol, Amelia Bilbao

Terradas, Jaume

Tervahauta, Taina

Tessler, Zachary

Testa, Riccardo

Testi, Daniele

Tetu, Amelie

Teuber, Ramona

Thakur, Sudhir K.

Thakur, Vijay Kumar

Thanopoulou, H. A.

Thanos, Sotirios

Thappa, Rajesh Bahadur

Themistocleous, Kyriakos

Theodoridis, Evangelos

Theodorou, Maria

Theuerkauf, Jörn

Theurl, Michaela

Thielen, Clemens

Thilmany, Dawn

Thirugnanam, Kannan

Thomas, Andrew

Thomas, Deborah S.K.

Thomas, Hywel

Thompson, Carol

Thompson, Fred

Thorarinsdottir, Ragnheidur

Thorleuchter, Dirk

Thorpe, David Stuart

Thorsen, Andreas

Thulasiram, Ruppa K.
Thyberg, Krista

Tian, Zhenhua

Tiari, Saeed

Tilt, Bryan

Tilt, Carol

Tinnilä, Markku

Tipi, Nicoleta

Tiranti, Davide

Tischer, Alexander

Tobias, Silvia

Tobita, Hiroyuki

Tochkov, Kiril

Todd, Rebecca

Todd, Stephen

Todeschini, Sara

Todhunter, Paul E.

Todorova, Tamara

Toftum, J.

Tokatli, Nebahat

Tokgöz, Emre

Tokuhiro, Akira

Tolonen, Anja

Toloo, Mehdi

Tomlinson, Douglas

Tondelli, Simona

Tonelli, Flavio

Tong, Daoqin

Tonjes, David J.

Tonmoy, Fahim

Tonosaki, M.

Toon, Richard

Toparlar, Y.

Toppinen, Anne

Torbert, Alen

Tordeux, Antoine

Torero Cullen, Jose

Torok, Adam

Toro-Mujica, Paula

Torre, Salvo

Torres, Luis

Torres Camacho, J.N.

Torresi, Marco

Torres-Machi, C.

Torricelli, C.

Torrisi, Gianpiero

Torvanger, Asbjørn

Totten, Jeffrey W.

Touhy, Paul

Toyasaki, Fuminori

Trammell, E. Jamie

Tran, Chinh C.

Trapani, Kim

Traverso, Marzia

Tredici, Peter Del 
Trenchard, Liz

Trencher, Gregory

Trianni, Andrea

Triantakonstantis, D.

Trifkovic, Neda

Trigunarsyah, B.

Trivess, Moore

Trögl, Josef

Tronchin, Lamberto

Trulli, Ettore

Truninger, Mónica

Trupp, Alexander

Tsagarakis, Konstantinos

Tsai, Chen-Tsang (Simon)

Tsai, I-Chun

Tsai, Kang-Ting

Tsai, Sang-Bing

Tsai, Wen-Hsien

Tsang, Dan

Tsangaratos, Paraskevas

Tsartas, Paris

Tscharaktschiew, Stefan

Tse, Mike

Tselios, Vassilis

Tseng, Ming-Lang

Tsiakas, Ilias

Tsikaloudaki, Katerina

Tsolakis, Naoum

Tsunekawa, Atsushi

Tudor, Terry

Tuladhar, Arbind

Tulloch, Lynley

Turner, Brian

Turner, John A.

Tyfield, David

Tzeng, Gwo-Hshiung

Tziourtzioumis, Dimitrios

Tzortzopoulos, Patricia

Udo, Henk

Ugarte, Carmen M.

Uhlaner, Lorraine M.

Ulanowicz, Robert

Uleberg, Eivind

Ulibarri, Nicola

Ülkü, M. Ali

Ullrich, Torsten

Ulrich-Schad, Jessica

Umeda, Yasushi

Unami, Koichi

Unver, Olcay

Uprety, Yadav

Ur Rahman, Saleem

Urken, Arnold B.

Urli, Tommaso
Usher, Lindsay

Uslar, Mathias

Uusitalo, Ville

Vaidya, Ashma

Väisänen, Maria

Vajčnerová, Ida

Valada, Tatiana

Valente, Clara

Valente, Mike

Valero-Verdú, Sergio

Validi, Sahar

Valko, Orsolya

Valkokari, Katri

Vallet, Flore

Valls-Donderis, Pablo

Valverde, Raul

Valverde, Raul

Van Dam, Jan E.G.

Van Dam, Pieter

Van De Ven, Frans

Van Den Belt, Henk

Van Den Hurk, Bart

Van Den Hurk, Martijn

Van Der Linden, Sander

Van Der Sijde, Peter

van der Werf, Edwin

Van Der Werff, Ellen

Van Kerkhoff, Lorrae

van Koppen, C.S.A. (Kris)

Van Loon, Patricia

Van Putten, Ingrid

Van Raaij, Fred

Van Vliet, Jasper

Van Vliet, Oscar

Van Wee, Bert

Van Zyl, Dirk

Vanbroekhoven, Karolien

Vandaele, Nico J.

Vandecasteele, Bart

Vanhonacker, Filiep

Vanolo, Alberto

Varela, Elsa

Varela, M.L.R.

Varela-Candamio, Laura

Varotsos, Costas

Varsei, Mohsen

Vasilakos, Athanasios V.

Vasquez, Alejandro

Vasseur, Véronique

Vaz, Deisi Altmajer

Vaz, Pedro G.

Vazquez, Angela

Vázquez-Rowe, Ian

Vedantam, Aditya
Veeck, Gregory

Vega-Marcote, Pedro

Velázquez, Suzanne L.

Vellema, Sietze

Velpuri, Naga Manohar

Velten, Sarah

Vemury, Chandra Mouli

Vera Rebollo, José Fernando

Verbas, Omer

Verbruggen, Aviel

Verdelhos, $\mathrm{T}$.

Verginelli, Iason

Verhoeven, Geert

Versini, P.-A.

Verticale, Giacomo

Vezzetti, Enrico

Viana, H.F.D.S.

Viassone, Milena

Vicinanza, Diego

Vickery, Jonathan

Viere, Tobias

Vignudelli, Stefano

Vij, Akshay

Villanueva-Rey, Pedro

Villarroya, Ana

Villasante, Sebastian

Villaverde, Juan José

Vincenzo, Torretta

Vinci, Ignazio

Vinke-de Kruijf, Joanne

Viotti, Paolo

Visaria, Sujata

Vishnivetskaya, Tatiana A.

Vitale, Pierluca

Vittuari, Matteo

Vittucci, Cristina

Vivarelli, Marco

Viveros, Hector

Viviano, Antonino

Vizzari, Marco

Vlachogiannis, D.

Vlachos, Dimitrios

Vladimirova, Katia

Vlajic, Jelena

Vlontzos, George

Vo, Minh D.

Vogelmann, James

Voigt, Christian

Volland, Gerhard

Vollmer, Hendrik

Volos, Christos

Voroney, Paul

Vosough Ahmadi, Bouda

Vossen, Jack H. 


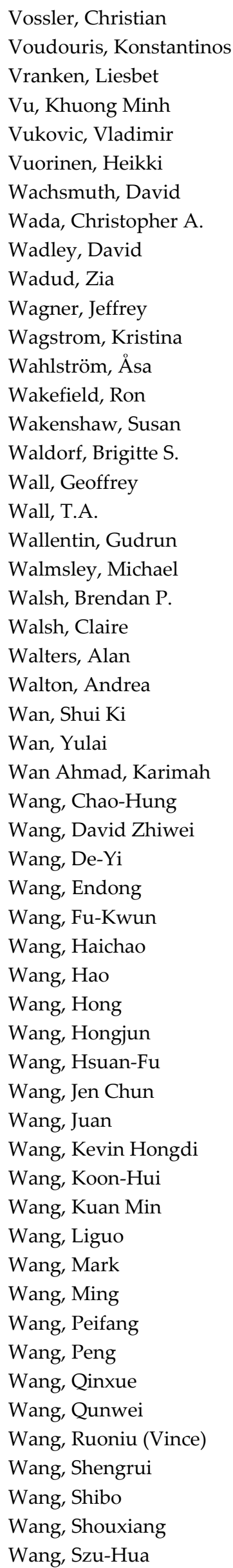

Wang, Tong

Wang, William Y.C.

Wang, Xia

Wang, Yachan

Wang, Yubo

Wang, Yupeng

Wang, Zeyu

Wanner, Thomas

Ward, Peter Michael

Ward, Timothy

Wargocki, Pawel

Warner, Doug

Warren-Myers, Georgia

Watanabe, Wako

Watmough, Gary

Weatherley, Tony

Webb, Ashley

Weber, Bernd

Weber, Christiane

Weber, Olaf

Wehrden, Henrik Von

Wei, Bing

Wei, Chu

Wei, Guiwu

Wei, Xiupeng

Wei, Yehua Dennis

Weichhart, Georg

Weikard, Hans-Peter

Weil, Raymond

Weiller, Claire

Weimin, Huang

Weisstein, Fei L.

Wells, E. Christian

Wells, Peter

Welz, Juliane

Wen, Chieh-Hua

Wen, Tzai-Hung

Wenger, Robert B.

Werner, Gerwin

Wesener, Andreas

Wesner, Jeff

Wesseler, Justus

Wesseling, Joeri

Wesselink, Renate

West, Paul C.

Westerlund, Mika

Westerman, Wim

Whatley, Art A.

Wheeler, Stephen

Whelan, Christopher

While, Aidan

White, Angela M.

White, Greg

White, Michael
Whitehouse, Hilary

Whittington, Dale

Whitty, Mark Albert

Wibowo, Santoso

Wichelns, Dennis

Wiedenhofer, Dominik

Wiering, Mark

Wiese, Frauke

Wiesmeier, Martin

Wilhelmsson, Mats

Wilkes, Martin

Wilkins, Ross

Wilkinson, Mark

Will, Matthias Georg

Willaarts, Barbara

Willis, J. Richard

Willison, Martin

Wilson, Jessica Marie

Winson-Geideman, K.

Winter, Jennie

Wirl, Franz

Wise, Nicholas

Wiseman, Alexander

Wisner, Ben

Witlox, Frank

Wittmayer, Julia Maria

Wittwer, Glyn

Wojtkowska, Małgorzata

Woldesenbet, Asregedew

Wolf, Steven

Wolfe, Philip J.

Wolsink, Maarten

Wolstenholme, J.K.

Wolszczak-Derlacz, J.

Won, Kwangjai

Wong, Ling-Tim

Wong, Sam

Wong, YingQi

Woo, Nam Chil

Wood, Benjamin

Wood, Emma

Wood, Michael

Wood, Roy C.

Woodley, Alan

Woodman, Bridget

Wooldridge, Christopher

Woolvin, Amy

Worm, Jeremy

Wouter, De Groot

Wray, Meredith

Wreford, Anita

Wright, Glen

Wright, Mark Mba

$\mathrm{Wu}$, Changshan 
Wu, Chih-Wen

$\mathrm{Wu}, \mathrm{Hao}$

Wu, Hong

$\mathrm{Wu}$, Jianshuang

$\mathrm{Wu}$, Jixiang

$\mathrm{Wu}$, John

Wu, Pei-Ing

Wu, Qiusheng

$\mathrm{Wu}$, Ruidong

$\mathrm{Wu}$, Tsaur-Chin

$\mathrm{Wu}$, Wei

$\mathrm{Wu}, \mathrm{Wei}$

$\mathrm{Wu}$, Zhengping

Wuest, Thorsten

Wüpper, David

Xenidis, Yiannis

$X i$, Lin

Xia, Jianhong (Cecilia)

Xiao, Yi-Yong

Xie, Hongjie

Xie, Ning

Xie, Xiaoping

Xie, Yichun

Xie, Yingying

Xing, Ke

Xiong, Yongzhu

$\mathrm{Xu}$, Jialin

$\mathrm{Xu}, \mathrm{Li}$

Xu, Li Da

$\mathrm{Xu}$, Tianfang

$\mathrm{Xu}$, Xiandong

Xu, Yongming

Xu, Zengwang

$\mathrm{Xu}, \mathrm{Zhi}$

Xuan, Jin

Xue, Bing

Xylia, Maria

Yagi, Michiyuki

Yalabik, Zeynep Yesim

Yan, Hong

Yan, Xing

Yang, Chenlung

Yang, Chia-Han

Yang, Chi-jen

Yang, Dujuan

Yang, Eric (Xue-Ming)

Yang, Hairui

Yang, Hengzhao

Yang, Jiachuan

Yang, Jinyan

Yang, Kuo-Lung

Yang, Ming

Yang, Rebecca

Yang, Wei
Yang, Xining

Yang, ZaiLi

Yang, Zan

Yang, Zijiang

Yao, Chen-Yen

Yao, Jing

Yazan, Devrim

Yazdani, Morteza

Yazici, M. Anil

Ye, Bin

Ye, Zhisheng

Yeboah-Forson, Albert

Yeh, Chia-Tsung

Yeh, Ching-Chiang

Yeh, Sonia

Yeh, Wei-Chang

Yen, Barbara T.H.

Yen, Neil Y.

Yen, Tian Ming

Yengoh, Genesis

Yeo, Hwasoo

Yeo, Zhiquan

Yepes, Víctor

Yezer, Anthony

Yi, Hongtao

Yi, June-seong

Yi, Pengxing

Yi-Kai, Juan

Yilmaz, Kuzey

Yin, Huajun

Yin, Peng-Yeng

Yin, Runsheng

Ying, Fei

Yokozawa, Masayuki

Yoo, Do Guen

Yoo, Ga Young

Yoshida, Aya

Youdelis, Megan

Young, Sean

Younus, Md

Youssef, Ahmed M.

Yu, Bailang

Yu, Chia-Yuan

$\mathrm{Yu}, \mathrm{Hao}$

$\mathrm{Yu}$, Vincent F.

Yu, Youngjin

Yuan, Chao

Yuan, Hongping

Yuan, J.H.

Yuan, Yihong

Yuan, Zengwei

Yubero, E.

Yue, Yaojie

Yuen, Richard Kwok Kit
Yuichi, Hyakawa

Yun, Hayong

Yung, Winco K.C.

Yau, Y.

Yusuf, Wie

Zaccour, Georges

Zacharof, Myrto-Panagiota

Zaki, Mohamed

Zakšek, Klemen

Zaleski, Halina

Zamagni, Alessandra

Zaman, Atiq Uz

Zambrano González, Luis

Zamfir, Andreea

Zander, Kerstin K.

Zanon, Bruno

Zarei, Mohammad Hossein

Zarkadas, Ioannis

Zavadskas, E.K.

Zavala-Araiza, Daniel

Závodská, Anita

Zawidzki, Machi

Zea Escamilla, Edwin

Zecha, Stefani

Zeeman, Grietje

Zeng, Shihong

Zeng, Wente

Zeng, Yaohui

Zerbe, Stefan

Zevenbergen, Chris

Zhang, Chuanguo

Zhang, Da

Zhang, Desheng

Zhang, Guoxing

Zhang, Hao

Zhang, Heng

Zhang, Hui

Zhang, Jie

Zhang, Lin

Zhang, Ni

Zhang, Ning

Zhang, Pengfei

Zhang, Qian Forrest

Zhang, Qichun

Zhang, Qingyuan

Zhang, Rongpeng

Zhang, Wenzhong

Zhang, Xiantao

Zhang, Xiaoshuan

Zhang, Xin

Zhang, Yingfeng

Zhang, Yongli

Zhang, Yuanzhi

Zhang, Yuejun 
Zhang, Yulong

Zhang, Zhao

Zhang, Zhiqiang

Zhang, Zuo-Peng

Zhao, Dengji

Zhao, Gang

Zhao, Hengbing

Zhao, Peng

Zhao, Rui

Zhao, Simon Xiaobin

Zhao, Tian

Zhao, Xianbo

Zhao, Xianhui

Zhao, Xueting

Zhao, Yushan

Zhao, Zhan

Zhao, Zhiming

Zheng, Zeyu
Zhong, Liheng

Zhou, Chuanbin

Zhou, Jian

Zhou, Peng

Zhou, Tianying

Zhou, Yanlian

Zhou, Yutian

Zhou, Yuyu

Zhu, Bangzhu

Zhu, Huayang

Zhu, Jianting

Zhu, Jie

Zhu, Songli

Zhu, Yifang

Zhu, Yunxia

Zhu, Zhe

Ziegler, Friederike

Zikeli, Sabine
Zimmermann, Albert

Zobel, Christopher

Zoppi, Corrado

Zoras, Stamatis

Zou, Yonghua

Zsifkovits, Helmut

$\mathrm{Zu}$, Xingxing

Zuidwijk, Rob

Zullo, Francesco

Zumbrunnen, David A.

Zuniga-Teran, Adriana

Zurayk, Rami

Zurbruegg, Christian

Zurbrügg, Christian

Zutshi, Ambika

Zvezdov, Dimitar

(C) 2017 by the authors; licensee MDPI, Basel, Switzerland. This article is an open access article distributed under the terms and conditions of the Creative Commons Attribution (CC-BY) license (http://creativecommons.org/licenses/by/4.0/). 